\title{
L'inclassable persécution des Juifs.Quand les autorités belges à Londres préparaient le jugement des crimes de guerre allemands
}

Marie-Anne Weisers

\author{
(2) OpenEdition \\ Journals \\ Édition électronique \\ URL : http://journals.openedition.org/cmc/695 \\ DOI : $10.4000 / \mathrm{cmc} .695$ \\ ISSN : 2684-3080 \\ Éditeur \\ Fondation de la Mémoire Contemporaine
}

Édition imprimée

Date de publication : 1 décembre 2008

Pagination : 99-138

ISSN : 1377-1256

Référence électronique

Marie-Anne Weisers, «L'inclassable persécution des Juifs.Quand les autorités belges à Londres préparaient le jugement des crimes de guerre allemands », Les Cahiers de la Mémoire Contemporaine [En ligne], 8 | 2008, mis en ligne le 01 février 2020, consulté le 14 novembre 2020. URL : http:// journals.openedition.org/cmc/695; DOI : https://doi.org/10.4000/cmc.695 


\section{L'inclassable persécution des J uifs Quand les autorités belges à L ondres préparaient le jugement des crimes de guerre allemands}

\section{Marie-Anne W eisers}

La préparation du jugement des criminels de guerre par les Alliés pendant la Seconde Guerre mondiale a été au cœur d'études aussi nombreuses qu'éclairantes1. Or on sait fort peu de choses de la contribution belge à cet intense débat mené dans le milieu des gouvernements en exil à Londres. L es historiens mettent particulièrement l'accent sur le procès de Nuremberg et l'action préalable de la U nited $\mathrm{N}$ ations $\mathrm{W}$ ar Crimes Commission, tandis que les spécialistes de droit pénal international apportent de précieux éléments sur le rôle joué par d'autres intervenants qui précédèrent la création de la U NW CC en 19432.

D'autres organismes, en particulier l'I nternational Commission on $\mathrm{Pe}$ nal Reconstruction and Development (ou Commission de Cambridge) et la L ondon I nternational A ssembly, ont fourni un travail préalable et même fondateur à plusieurs égards. Des B elges désignés par le gouvernement en exil à L ondres y ont joué un rôle moteur, en contraste avec la préparation pour le moins parcellaire par ce même gouvernement de la répression des crimes de guerre au niveau national. Le gouvernement se montre par contre très actif en ce qui concerne la préparation de la répression de la collaboration. Une série de dispositions législatives sont prises dans ce domaine, mais aucune n'incrimine les mesures spécifiques prises à l'égard des J uifs. Quant à l'unique arrêtéloi établi par le gouvernement Pierlot, le 5 août 1943, lié à la problématique des crimes de guerre allemands, il ne sanctionne pas non plus les persécutions antijuives, pas plus qu'elles ne

\footnotetext{
${ }^{1}$ Cette recherche a bénéficié du financement de la $F$ ondation pour la Mémoire de la Shoah ( $P$ aris). J e remercie également les professeurs É ric David et Pieter Lagrou pour leurs précieux commentaires et les fructueuses discussions que je leur dois.

2 Pour les historiens: D. Bloxham, Genocide on Trial : W ar Crimes Trials and the $\mathrm{F}$ ormation of $\mathrm{H}$ olocaust $\mathrm{H}$ istory and M emory, Oxford, 2001; A. J . K ochavi, Prelude to $\mathrm{N}$ uremberg: Allied W ar Crimes Policy and the Question of P unishment, Chapel Hill, 1998; B. Smith, The Road to N uremberg, N ew Y ork, 1981. Pour les juristes: B. B. Ferencz, An International Criminal Court, a Step T oward W orld P eace: A D ocumentary H istory and A nalysis, New-Y ork, 1980 ; T. L. H. McCormack - G. J. Simpson (éds.), T he L aw of W ar Crimes, $\mathrm{N}$ ati onal and I nternational A pproaches, La H aye, 1997.
} 
sont évoquées dans le rapport au conseil des ministres établi par le ministre de la J ustice qui précède le texte de loi3.

A insi, invité en juillet 1943 à une séance de l'I nternational Commi ssion on $P$ enal R econstruction and D evelopment à s'exprimer sur ce que pensent les B elges "de l'intérieur", A ntoine Delfosse, ministre de la J ustice et de I'I nformation du gouvernement belge en exil à L ondres, explique qu'à son sens « le pays occupé réclame un jugement rapide par les juridictions nationales et l'exécution effective et complète des peines $\gg 4$. En réalité, la justice belge d'après-guerre ne procèdera ni à un jugement rapide des criminels de guerre allemands, ni à une exécution effective et complète des peines prononcées. Les procès ne débuteront qu'en août 1948 et, à I'exception des cas d'E duard Strauch (chef de la Sicherhei tspolizei [Sipo] de L iège et d'A rlon, mort en prison en 1956), de Philip Schmitt (chef du camp de B reendonk de septembre 1940 à novembre 1943 et de la caserne D ossin à Malines de juillet 1942 à mars 1943, exécutéle 8 août 1950) et de Walter Obler (J uif autrichien, «chef de chambre» au camp de Breendonk, exécuté le 12 avril 1947), tous les prisonniers allemands seront "extraits" de prison et reconduits à la frontière allemande en 1952 au plus tard5.

L e cas belge n'est pas unique. II s'inscrit dans un contexte général de guerre froide unissant A méricains et $E$ uropéens dans une lutte commune contre le bloc communiste. Ce combat nécessite une $E$ urope forte incluant obligatoirement I'Allemagne fédérale. À partir de 1947, les alliés américains et anglais commencent à démanteler leur programme de jugement des criminels de guerre, ce qui ne manquera pas d'avoir un impact sur les poursuites exercées par la B elgique, puisqu'ils décident l'arrêt des extraditions des ressortissants allemands dès mars 19486. E n 1949, lors de la créa-

\footnotetext{
3 Moniteur bel ge, 20 septembre 1943.

${ }^{4}$ Sur l'I nternational Commission, voir infra. Antoine D elfosse, docteur en droit et en philologie classique de l'U niversité de L iège, est démocrate-chrétien. R ésistant, il devient entre 1942 et 1944 ministre de la J ustice et de l'I nformation du gouvernement en exil à L ondres, puis en décembre 1944 président de la Commission des Crimes de Guerre. Pour sa déclaration : Centre d'É tudes et de Documentation Guerres et Sociétés contemporaines (CEGES), AA 418, A rchives Inbel, 1003, discours à la R adio nationale belge de L ondres, 9.10.1943.

5 Sur Obler : P. N efors, B reendonk 1940-1945, B ruxelles, 2005, pp. 264-266.

${ }^{6} \mathrm{P}$. Lagrou, « E ine $\mathrm{F}$ rage der moralischen Überlegenheit ? Die A hndung deutscher $\mathrm{K}$ riegsverbrechen in B elgien", dans N. F rei (éd.), Transnationale Vergangenheitspolitik. Der U mgang mit deutschen $\mathrm{K}$ riegsverbrechern in E uropa nach dem Zweiten W eltkrieg, Göttingen, 2006, p. 334 ; N. Wouters, « La persécution des J uifs devant les juges belges (1944-1951) », dans R. Van Doorslaer (éd.), L a B elgique
} 
tion de l'Allemagne fédérale et de sa reconnaissance par la Belgique, les pressions allemandes, et notamment celles exercées par le chancelier K onrad Adenauer, se feront de plus en plus pressantes pour que le gouvernement libère les prisonniers allemands?.

$L$ a B elgique va toutefois se différencier des autres pays, notamment de la F rance - voisin traditionnellement de référence en matière de droit -, d'une part, par sa lenteur à se doter d'une loi applicable en matière de crimes de guerre et, d'autre part, par la manière d'incriminer les actes commis par l'occupant allemand.

L a loi belge sur la compétence des juridictions militaires en matière de crimes de guerre est en effet promulguée le 20 juin 1947, alors que I'ordonnance française date déjà du 28 août 1944. Quant aux différences de fond, le législateur belge prévoit que l'infraction est constitutive de crime de guerre si elle répond à une double condition : constituer une infraction prévue par la loi pénale belge et une atteinte aux lois et coutumes de la guerre. L'infraction est donc à la fois d'ordre interne et d'ordre international. L'ordonnance française du 28 août 1944 relative à la répression des crimes de guerre simplifie par contre fortement les choses : elle se délie des contraintes du droit international et définit le crime de guerre comme étant un crime de droit commun commis en temps de guerres. Autre distinction majeure: le texte français contourne l'obstacle de l'atteinte au principe de non-rétroactivité des lois en procédant à une interprétation d'autorité de certaines dispositions du Code pénal et du Code de justice militaire. C'est ainsi que l'article 3 de l'ordonnance française assimile « toute exposition dans les chambres à gaz » à l'empoisonnement prévu à l'article 301 du Code pénal ; de même, elle assimile la déportation et le travail obligatoire à la séquestration prévue aux articles 341 et suivants du code pénal.

L es auteurs de la loi belge, critiques à l'égard de ce procédé, ont refusé d'y recourir, privilégiant le pouvoir d'interprétation du juge, qui s'avèrera totalement insuffisant pour pallier les carences du code pénal en la ma-

docile. Les A utorités belges et la persécution des J uifs en B elgique durant la Seconde Guerre mondiale, B ruxelles, 2007, pp. 982-985.

${ }^{7}$ Archives du M inistère des Affaires étrangères (AMAE) ), dossier 12.402, passim.

8 C. Moisel, «Des crimes sans précédent dans I'histoire des pays civilisés : I'occupation allemande devant les tribunaux français, 1944-2001 », dans G. E ismann - S. Martens (éds.), Occupation et répression militaire allemandes 1939-1945: la politique de "maintien de l'ordre" en E urope occupée, Paris, 2007, p. 189. 
tière. À l'instar de toutes les législations de l'époque, le code pénal belge ne contient aucune disposition qualifiant les faits de déportation, persécution ou exécution d'otages. Faute de dispositions ad hoc, et malgré des efforts d'imagination, les juges belges ne parviendront pas à inculper les criminels de guerre allemands pour la perpétration de crimes jusqu'alors méconnus et commis en dehors de tout sens commun.

Pour pouvoir apprécier correctement le rôle des intervenants belges dans la préparation de la répression des crimes de guerre, tant au niveau international que national, il est indispensable de décrire la faillite des poursuites pour crimes de guerre après la P remière Guerre mondiale, qui a marqué les esprits. Mais il convient aussi que le lecteur garde à l'esprit, d'une part, les contraintes que rencontre le chercheur dans ce domaine et, d'autre part, deux facteurs particuliers qui ont conditionné le cas belge.

Le chercheur qui veut analyser les archives relatives à la préparation législative du jugement des crimes de guerre doit faire face aux nombreuses carences qui caractérisent ces archives. Elles sont tout à la fois dispersées, parcellaires, indisponibles, voire inexistantes dans certains cas. Contrairement aux archives du ministère des Affaires étrangères, les archives du ministère de la J ustice ne sont pas centralisées et il faut donc procéder au coup par coup. Or, de façon surprenante, les papiers personnels des ministres de la J ustice qui se sont succédé de 1945 à 1947 ne contiennent aucun document concernant la problématique de la répression des crimes de guerre. Quant aux archives personnelles d'Antoine D elfosse, personnage clef de l'époque, puisqu'il sera d'abord ministre de la J ustice de 1942 à 1944, puis président de la Commission belge des Crimes de Guerre en 1945, elles sont pour le moment inaccessibles au public. II en va de même des papiers de $F$ rédéric Dumon, substitut du procureur du roi lors des procès d'après-guerre et collaborateur de Walter Ganshof van der Meersch, auditeur général et haut-commissaire à la Sûreté de l'É tat en exil à Londres.

Les deux facteurs, bien connus, relèvent de la problématique de la ré pression belge en général. Celleci est centrée au niveau politique et judiciaire sur la répression des collaborateurs, des «traîtres à la patrie». Les autorités accordent une priorité absolue à la poursuite des Belges ayant porté atteinte à la sûreté extérieure de l'É tat. Les crimes commis par les étrangers allemands n'occupent qu'une place secondaire, alors même qu'ils sont les initiateurs de la politique criminelle en pays occupé, dans 
certains cas les donneurs d'ordre, dans d'autres les auteurs directs des faits commis.

Par ailleurs, la poursuite des actes criminels relatifs à la politique antijuive instaurée par l'occupant est quasiment occultée. La question de savoir comment sanctionner les auteurs de crimes commis contre les J uifs ne se pose qu'à de très rares occasions et ne fait I'objet d'aucun traitement spécifique. Par contre, les archives contiennent de nombreux documents relatifs à la situation des J uifs en Belgique. Même si certaines informations sont parfois fausses ou contradictoires, fin de l'année 1942, le gouvernement belge à Londres a connaissance d'une série d'informations dramatiques sur le sort des J uifs, en ce compris un rapport faisant mention expresse de l'ordre d'extermination venu de Berlin'. Celles-ci devraient suffire pour justifier à tout le moins un questionnement sur la mise en place de mesures législatives aptes à poursuivre les auteurs de ces crimes nouveaux. Or les indications sur cette question précise sont absentes des archives consultées, qu'il s'agisse de documents couvrant la période de la guerre ou des archives parlementaires des années 1945 à 1947 portant sur les projets de loi relatifs à la compétence des juridictions en matière de crimes de guerre. $E n$ matière de sanction, le débat porte plutôt sur la question des otages que sur la question juive et la déportation.

\section{Les crimes de guerre et la Première Guerre mondiale}

Pour comprendre la problématique du jugement des criminels de guerre telle qu'elle se pose durant la période étudiée, il faut remonter à la P remière Guerre mondiale et à l'échec des procès qui s'en sont suivis. L ors de la Conférence de la Paix du 18 janvier 1919, les gouvernements alliés décident la création de diverses commissions, dont l'une est chargée d'examiner les responsabilités des auteurs de la guerre, les infractions aux

\footnotetext{
${ }_{9}$ AMAE, dossier 11.742, note s.n., s.l., "German Persecution of the J ews in Occupied Belgium », 19.12.1942 (trad. : "La persécution des I sraélites en Belgique occupée », 30.12.1942). Voir V. Laureys, " $L$ 'attitude du gouvernement belge en exil à $L$ ondres envers les J uifs et la question juive pendant la Seconde Guerre mondiale», dans R. Van Doorslaer (éd.), Les J uifs de Belgique. De l'immigration au génocide, 1925-1945, B ruxelles, 1994, pp. 137-152. P our une étude approfondie de la connaissance par le gouvernement belge en exil à Londres du sort réservé aux J uifs de Belgique, voir E. D ebruyne, «F ace à une impensable extermination (été 1942 - été 1944) », dans R. Van D oorslaer (éd.), L esJ uifs de B elgi que..., op. cit., pp. 709-810.
} 
lois et coutumes de la guerre, ainsi que la possibilité d'établir un tribunal qui puisse connaître de tels crimes. Deux mois plus tard, la Commission on the R esponsability of the $A$ uthors of the $W$ ar and on $E$ nforcement of $P$ enalties dénonce dans son rapport du 29 mars 1919 le déclenchement d'une guerre d'agression d'abord par l'Allemagne et l'A utriche, puis par la Turquie et la Bulgarie. Elle établit une liste de trente-deux actes constituant des violations flagrantes des lois et coutumes de la guerre et des lois d'humanité. Sont citées entre autres atrocités : les meurtres et massacres massifs de civils et de prisonniers de guerre, les tortures, l'exécution d'otages, le viol, le pillage, la destruction arbitraire de propriété publique ou privée, les punitions collectives, l'usage de gaz toxiques'10. La Commission recommande aussi la mise en place d'un haut tribunal compétent pour juger de ces crimes selon « les principes des lois des nations tels qu'ils résultent des usages établis parmi les peuples civilisés, des lois d'humanité et des exigences de la conscience publique ${ }^{11}$. Elle souhaite enfin que les gouvernements des pays alliés adoptent cette législation et fassent le né cessaire pour assurer la mise en œuvre des sentences rendues.

Mais les A méricains et les J aponais vont émettre des réserves importantes sur le rapport, notamment sur l'aspect trop vague des lois et coutumes de la guerre et sur le fait que des crimes nouveaux (tel que le crime d'agression), soumis à cette juridiction nouvelle selon des lois nouvelles présenteront un caractère rétroactif qui contrevient à la Constitution américaine ${ }^{12}$. En fait, peu de gouvernements appliqueront ces recommandations et, en fin de compte, le tribunal international ne verra pas le jour. Le traité de Versailles est signé au mois de juin et prévoit dans ses articles 228 et suivants que les Allemands accusés d'avoir violé les lois et coutumes de la guerre seront livrés aux pays alliés et jugés devant leurs juridictions militaires. Une liste des criminels de guerre est établie, mais I'Allemagne parviendra à convaincre les gouvernements alliés d'accepter que les inculpés soient déférés non devant les tribunaux militaires des différents pays alliés, mais devant ses propres juridictions, en l'occurrence

\footnotetext{
10 Le texte du rapport de la Commission on the Responsability of the A uthors of the War and on the E nforcement of $P$ enalties est reproduit intégralement dans B. B. Ferencz, A n International Criminal Court..., op. cit., pp. 169-192.

11 | bid., p. 182.

12 T. L. H. McCormack, «From Sun Tzu to Sixth Committee: The Evolution of an International Criminal Law Regime», dans T. L. H. McCormack - G. J . Simpson (éds.), The Law of W ar Crimes..., op. cit., p. 47.
} 
la Cour suprême de Leipzig. Les procès de Leipzig, qui débutent en mai 1921, seront par la suite qualifiés de farce judiciaire: les accusés auront soit disparu, soit été acquittés pour preuves insuffisantes ou controversées. Les plus indignés seront les gouvernements français et belge, repré sentants des pays dont les populations ont le plus souffert de l'occupation allemande : travail forcé pour l'effort de guerre ennemi, évacuations forcées, déportation, prises $\mathrm{d}^{\prime}$ otage $^{13}$. Dans le cas belge, sur une liste initiale de 1.132 criminels de guerre dont l'extradition est réclamée par les autorités, quinze dossiers seulement seront présentés devant la Cour de L eipzig et cinq feront l'objet d'une commission rogatoire du procureur général allemand, qui finalement ne retiendra aucune charge ${ }^{14}$.

Cet échec du jugement des criminels de guerre après 1918 sera lourd de conséquences et le souci de ne pas le répéter constituera une obsession chez ceux qui, de 1941 à 1945, prépareront le jugement des criminels de guerre de la Seconde Guerre mondiale.

Durant l'entre-deux-guerres, parallèlement à l'adoption de deux Conventions à Genève en 1929 sur le traitement des prisonniers de guerre et pour l'amélioration du sort des blessés et des malades dans les armées en campagne, des démarches seront entreprises et des appels lancés pour tenter de mettre sur pied un tribunal international et d'établir un code de droit international, mais sans que cela n'aboutisse à de grands résultats. Le manque de cohésion et de volonté de la communauté internationale en est bien sûr l'une des causes principales. Si elles n'ont pas abouti, ces démarches ont toutefois fait évoluer les conceptions: " H owever, in spite of the lack of consensus, it is also clear that the concept of individual culpability for the violation of an international crime was much more widely accepted than it had been pri or to W W I. F urthermore, the adopti on by the L eague of $\mathrm{N}$ ations of the Convention for the $\mathrm{P}$ revention and $\mathrm{P}$ unishment of T errorism demonstrated a willingness to extend the content of international criminal law beyond exclusively war crimes. These factors, combined with the widespread frustrations and dissatisfaction with the lack of effective war crimes trials after the conclusi on of W W I, paved the way for the international responses to the commi ssion of atrocities during the course of W W II . »15

\footnotetext{
13 A. Becker, Oubliés de la Grande Guerre. H umanitaire et culture de guerre, 1914-1948. P opulations occupées, déportés civils, prisonni ers de guerre, Paris, 1998, pp. 33-34.

14 Voir à ce sujet: P. L agrou, «E ine F rage... », op. cit., pp. 326-350.

15 T. L. H McCormack - G.J. Simpson, «The Law of W ar Crimes... », op. cit. , p. 55.
} 
On trouve une illustration des tergiversations, des divergences de vue et des tensions internationales de l'époque dans un dossier de la Croix$R$ ouge conservé au ministère des $A$ ffaires étrangères. II concerne la X VI e Conférence internationale de la Croix-R ouge de Londres, qui s'est tenue du 20 au 28 juin 1938.

En mars 1936, la L égation suisse en Belgique s'adresse à Paul van Zeeland, à l'époque premier ministre et ministre des Affaires étrangères, pour l'informer et lui demander son avis sur l'intention du Conseil fédéral de convoquer à Genève " dès que les conjectures seront plus favorables », une conférence diplomatique de la Croix-R ouge chargée d'étendre aux civils la Convention de Genève de 1929, qui porte uniquement sur la protection des blessés et malades combattants. L'acte final de la Convention de 1929 contenait le vœu de réunir une telle conférence. $L$ 'auteur de la lettre dé plore que «les circonstances ne se prêtent guère à une réunion de cette importance. Ce n'est pas dans une atmosphère de sanctions que l'esprit de collaboration internationale qui a caractérisé jusqu'ici les conférences diplomatiques de la Croix-R ouge pourrait mieux s'affirmer », mais ajoute que «les raisons qui avaient motivé, en 1929, l'adoption des vœux prérappelés subsistent plus que jamais. Qu'on le veuille ou non, la guerre reste dans l'ordre des possibilités. Mon gouvernement le regrette plus que personne, mais force lui est de regarder la réalité en face et, vu les risques de conflits armés qui continuent à peser sur le monde, de chercher à améliorer les instruments juridiques qui ont fait de la Croix-R ouge une institution universellement reconnue, appréciée et respectée $\gg 16$. La même communication est faite par la L égation suisse à la $\mathrm{H}$ aye, B erlin, L ondres, $P$ aris et $\mathrm{R}$ ome.

Cing mois plus tard, un membre du service juridique du ministère belge des Affaires étrangères rédige une note dans laquelle il constate « le peu d'empressement à donner suite en ce moment à l'invitation du gouvernement suisse. Cela ne nous surprend pas. La pensée dominante reste toujours que le moment n'est pas venu de s'occuper des lois de la guerre et qu'il faut s'occuper d'abord du désarmement. Personnellement, je ne partage pas cette manière de voir ; mais il ne servirait à rien d'aller de l'avant avec le gouvernement suisse, pour rester en l'air. On pourrait donc classer le dossier en attendant que son objet nous soit rappelé éventuellement. »17

\footnotetext{
${ }^{16} \mathrm{AMAE}$, dossier 12968, lettre de la L égation suisse en B elgique à $P$ aul van Zeeland, 03.03.1936.

17 l bid., noten ${ }^{\circ} 22279,31.08 .1936$.
} 
E n novembre de la même année, la L égation suisse informe Paul-H enri Spaak, nouveau ministre belge des Affaires étrangères, que l'Allemagne, I'I talie et les É tats-U nis d'A mérique ont donné un avis favorable à l'idée de convoquer une nouvelle conférence diplomatique de la Croix-R ouge, ce qui motive la B elgique à y répondre aussi favorablement 18 .

La Conférence internationale de la Croix-R ouge se tient un an et demi plus tard. Elle donne lieu à deux constats: tout d'abord, le nombre de points à améliorer n'est pas suffisant pour justifier une révision complète de la Convention de Genève du 27 juillet 1929 ; ensuite, certaines délégations suggèrent que la protection de la population civile ne fasse pas l'objet d'un projet de convention révisée, mais d'une convention particulière. E lle souhaite enfin la réunion d'une conférence diplomatique où les modifications proposées feront l'objet d'un protocole à annexer à la Convention de Genève, notamment la réunion en une seule convention de toutes les stipulations humanitaires ayant trait à la Croix-R ouge19.

En août 1939, le gouvernement suisse demande donc aux divers États leur avis sur l'organisation d'une nouvelle conférence. Le commentaire du juriste belge attaché au ministère des A ffaires étrangères est pour le moins déconcertant : "II semble bien qu'en ce moment il se produise un revirement quant à la nécessité d'un code des lois de la guerre. Les uns n'en voulaient plus par scepticisme quant à leur efficacité; d'autres n'en voyaient plus la nécessité, considérant la guerre comme abolie par les pactes de Genève et de Paris. Le gouvernement suisse $a$, il y a plusieurs semaines, rappelé sa proposition de réunir une conférence de révision des conventions dites "de la Croix-R ouge". I I ne semble pas, vu la situation actuelle, que cette idée soit réalisable en ce moment. II faudra attendre la fin de la guerre, de façon à pouvoir se rendre compte de l'étendue des lacunes à combler. $\gg 20$

Tout y est : les hésitations des décideurs, une prise de conscience chez certains, l'aveu d'inefficacité et finalement le report des choix. Toutefois, devant les atrocités sans précédent commises par les nazis, les chefs d'É tat n'attendront pas la fin de la guerre pour tenter de combler les nombreuses lacunes du droit.

\footnotetext{
18 I bi d. , lettre du Secrétaire général du ministère du Commerce extérieur à la L égation suisse en B elgique, 28.11.1936.

19 I bid., R ésolutions et vœux adoptés par la X VI e Conférence internationale de la Croix-R ouge, juin 1938.

20 | bid., note s.n, 25.09.1939.
} 


\section{Préparation internationale du jugement des criminels de guerre}

A u début de la guerre, la question du châtiment des criminels de guerre est évoquée par le gouvernement polonais en exil, mais sans que cela ne suscite vraiment de réaction de la part des Britanniques et des A méricains, qui préfèrent retarder autant que possible le règlement de cette question. C'est surtout sous la pression des gouvernements tchèque et polonais que ces derniers vont modifier leur attitude21.

Le 22 juin 1941, le plan B arbarossa est déclenché, l'armée allemande envahit le territoire soviétique et les troupes de la W ehrmacht, renforcées par les unités de la police et de la SS, procèdent à l'exécution des fonctionnaires du parti communiste et des partisans soviétiques, ainsi qu'à des fusillades en masse de la population juive.

Suite à la radicalisation de la guerre et à la possibilité dès l'automne 1941 que l'Allemagne perde la guerre, le premier ministre britannique Winston Churchill et le président américain $\mathrm{F}$ ranklin $\mathrm{D}$. R oosevelt font, le 25 octobre 1941, une déclaration commune, dans laquelle ils dénoncent les exécutions d'otages et les massacres commis en $\mathrm{F}$ rance, sans mentionner toutefois les tueries commises contre les I uifs. I Is avertissent leurs auteurs que la punition de ces crimes figurera parmi les buts principaux de la guerre22.

Vingt-six pays en guerre avec l'Allemagne et le J apon signent à Washington, le 1er janvier 1942, une déclaration par laquelle ils s'engagent à coopérer et à ne pas signer d'accord séparé avec les pays de l'A xe. C'est la première fois que le terme « $U$ nited $N$ ations » est utilisé ; il désignera par la suite l'action des organes interalliés avant de devenir l'appellation officielle du successeur de la Société des $\mathrm{N}$ ations23.

Le 13 janvier 1942, les représentants des gouvernements en exil signent à Londres la Déclaration de Saint-J ames. Y sont représentés la B elgique, la F rance, la Grèce, le L uxembourg, la N orvège, les $P$ ays-B as, la P ologne, la Tchécoslovaquie et la Yougoslavie; le gouvernement britannique n'accepte pas de faire partie des signataires. La déclaration constate le régime de terreur utilisé par l'Allemagne dans les pays occupés, mentionne

\footnotetext{
${ }^{21}$ A. J . K ochavi, op. cit., p. 3.

22 CE GES, A A 418, I nbel, 1335, 28.10.1941.

${ }^{23}$ B. B. F erencz, A n International Criminal Court..., op. cit., p. 57.
} 
les violations des Conventions de la Haye de 1907 et réitère l'avertissement de Churchill et R oosevelt selon lequel les responsables de ces violations devront répondre devant la justice de leurs crimes.

L es A méricains et les B ritanniques décident de coopérer avec les signataires de l'accord de Saint-J ames et annoncent dans une déclaration commune du 7 octobre la création d'une $F$ act $F$ inding Commission, chargée de rassembler les preuves des violations des lois internationales et de les communiquer aux différents gouvernements: la U nited $\mathrm{N}$ ation Commission for the Investigation of War Crimes. Celle-ci sera remplacée, le 20 octobre 1943, par la U nited N ati on W ar Crimes Commissi on (U NW CC).

L e 14 octobre 1942, les Soviétiques font une déclaration par laquelle ils soutiennent toutes les décisions prises par les Alliés, que ce soit en matière d'extradition, de coopération pour la recherche des criminels allemands, d'établissement d'un tribunal international et d'un code suffisamment sévère pour les punir24.

Le 1er novembre 1943, les États-U nis, la Grande-B retagne et I'UR SS signent par l'intermédiaire de leurs ministres des Affaires étrangères la Déclaration de Moscou, qui prévoit que les criminels de guerre allemands devront répondre de leurs actes dans les pays où les faits ont été commis, conformément aux lois en vigueur dans les pays respectifs. Les auteurs des crimes qui ne sont pas localisés géographiquement seront punis par une décision commune des gouvernements alliés.

À côté des déclarations politiques menaçant les responsables allemands de sanctions judiciaires, destinées aussi à impressionner l'opinion publique des pays occupés, des organes non gouvernementaux se mettent en place à Londres, dont l'objectif est de préparer l'après-guerre, notamment en matière de répression.

\section{International Commission for Penal Reconstruction and De- velopment}

Le premier organisme qui se consacre à cette tâche est I'I nternational Commission for P enal Reconstruction and D evelopment. II travaille en collaboration avec le D épartement de science criminologique de la Faculté de droit de Cambridge et organise une conférence le 14 novembre 1941 à

24 I bid., p. 59. Archives générales du Royaume (AGR), Commission pour l'Étude des Problèmes d'A près-guerre (CE PAG), 1523, extrait de $F$ rance du 1.04.1944. 
Cambridge, réunissant à la fois des ministres et des juristes britanniques et alliés. La B elgique est représentée par Albert De V leeschauwer, ministre des Colonies et ministre de la J ustice jusqu'en 1942, ainsi que par Marcel de Baer, président de la Cour militaire belge en Grande-B retagne. $Y$ assistent également le professeur R ené Cassin, commissaire national à la $J$ ustice de la F rance libre, et le professeur Stefan Glaser, ministre polonais auprès du gouvernement belge, deux juristes dont la renommée en matière de droit pénal international dépassait les frontières. La Commission se fixe pour mission d'établir une base légale à la répression et d'examiner les règles et procédures applicables aux crimes de guerre. À cet égard, elle préfère les qualifier de « crimes against I nternational Public Order » plutôt que de « war crimes, which in its popular sense would do well but in its technical sense would beinappropriate $\gg 25$.

Terminologie juridique, catégories juridiques et difficultés techniques sont les raisons maj eures et récurrentes de l'inaptitude du droit à répondre aux problèmes du moment, du décalage incessant entre la norme, ou l'absence de norme, et la réalité des faits.

Dans une lettre adressée au premier ministre H ubert Pierlot, relative à la préparation de la Conférence de Saint-J ames, A lbert De Vleeschauwer fait part de son mécontentement et s'interroge sur les questions de fonds :

«M on cher Premier Ministre,

J e reçois à l'instant le mot de $M$. Taymans me transmettant le projet de "Déclaration commune des Gouvernements [... ]".

$V$ oici les observations que la lecture attentive de ce texte $m$ 'inspire.

$1^{\circ} \mathrm{Un}$ accord de ce genre devrait être traité, je pense, par le D épartement des Affaires É trangères.

$2^{\circ} \mathrm{D}$ ans le texte de la note explicative à notre usage, il est dit que les violations des droits des gens, contre lesquelles on entend agir, n'ont rien de commun avec des actes de guerre ou des crimes politiques, et dans le texte du proj et on précise "que les violences qui à l'occasion de la guerre s'exercent actuellement contre les populations civiles, n'ont rien de commun avec l'acte de guerre ou le crime politique". J e me permets de faire observer que cette expression est loin d'être exacte et manque par trop de précision.

Dans la conférence de Cambridge à laquelle j'ai assisté vendredi dernier, j'ai prié l'assemblée d'étudier d'abord à fond cette même question du

25 CE GES, AA 658, Archives Albert De Vleeschauwer, I nternational Commission for P enal Law and Development, 14.11.1941. 
point de vue théorique avant d'y intéresser à un titre quelconque, les gouvernements politiques.

II se pose là, en effet, quatre questions très précises:

- quel crime entend-on poursuivre?

- devant quelle juridiction entend-on les poursuivre?

- quelles personnes seront considérées comme responsables de ces crimes?

- l'extradition sera-t-elle garantie par tous les pays signataires?

Le projet de déclaration actuel est en dessous de ce qui précède. J e crois franchement que sous l'aspect juridique il manque totalement de cette précision qui est nécessaire aux actes de ce genre. J 'ajoute qu'il serait d'ailleurs impossible à tout gouvernement d'être juridiquement précis en l'occurrence.

Dans le même texte de projet, dernier alinéa, ce manque de précision est vraiment trop grand. Les expressions qui se trouvent en note ne peuvent être ajoutées au dernier alinéa, car elles rendent le texte encore plus impossible. Au surplus, il y est question de solidarité internationale alors que les plus grandes nations ne participent pas à la déclaration. $\gg 26$

Le problème soulevé ici est de déterminer si ces actes seront considérés comme des crimes de droit commun relevant dès lors des juridictions nationales ou comme des infractions à caractère international nécessitant une législation internationale et la mise en place d'une juridiction internationale. La phrase décriée par De Vleeschauwer sera maintenue dans le texte de la Conférence interalliée. E lle implique que les actes commis sont considérés comme des crimes de droit commun.

Dans une lettre relative à la constitution éventuelle d'une Cour interalliée de J ustice, le directeur général du service politique du ministère des Affaires étrangères, faisant état de la question à l'administrateur de la Sûreté de l'É tat à Londres, lui fait part de son scepticisme quant aux chances de réussite du projet de la Commission de Cambridge: «I ndépendamment et en dehors de cette déclaration commune [il s'agit de la conférence de Saint-J ames], je sais qu'un mouvement se dessine à l'U niversité de Cambridge à l'effet de créer un droit pénal international européen en matière de crimes et délits de l'espèce visée. J e n'ai pas besoin de vous dire que cela apparaît comme une généreuse illusion et il suffira de rappeler à cet effet les tentatives poursuivies à la Société des Nations, lors de

26 | bi d. , lettre à H ubert Pierlot, 17.11.1941. 
I'assassinat du roi Alexandre 1er de Y ougoslavie, pour la création d'une Cour internationale deJ ustice pénale. ${ }^{27}$

Néanmoins, le travail accompli par la Commission fut très important et servit de base de réflexion aux autres organismes.

\section{London International Assembly}

Un deuxième organisme va jouer un rôle important dans la préparation de l'après-guerre: Ia L ondon International A ssembly, organe non officiel créé en 1941 sous l'égide de la L eague of $\mathrm{N}$ ations U nion et dont les membres sont nommés par les gouvernements alliés à Londres. Elle rassemble dix-sept États: aux neuf pays signataires de la Conférence de Saint-J ames s'ajoutent I'A frique du Sud, I'A ustralie, le Canada, les É tatsU nis, la Grande-B retagne, I'I nde, la Nouvelle-Zélande et I'U nion soviétique. L'objectif fixé est de mieux comprendre l'histoire, le développe ment économique, les institutions, le mode de vie, les aspirations de chaque nation et d'étudier les principes de la politique d'après-guerre et l'application de ces principes aux problèmes de reconstruction nationale et internationale ${ }^{28}$.

N euf commissions sont créées pour traiter ces différents sujets : Commission for Questions Concerned with the Liquidation of the War, Legal Commission, Commission for Collective Security and Disarmament, Commission on the Place of Education, Science and Learning in P ost-W ar Re construction, Commission for F inancial and E conomic Questions, Social and L abour Questions, Commission for International Organisation, Commission on the P lace of Religion in P ost-W ar R econstruction.

E $n$ juin 1943, une dixième commission est créée: The Commission on the J ewish Problem. Même si son instauration apparaît tardive, elle marque la volonté de la L ondon I nternational A ssembly de créer une commission centrée uniquement sur cette question, signifiant par là que le problème juif est pris en compte dans sa singularité. Toutefois, au début 1944, pour des raisons d'organisation interne, cette commission n'est toujours pas mise en place; d'abord, parce qu'il s'avéra compliqué de trouver

\footnotetext{
27 AMAE, dossier 11767, lettre du comte Charles de Romrée de Vichenet à Fernand Lepage, 28.11.1941.

${ }^{28}$ A GR , F onds J ef R ens, L ondon I nternational A ssembly, boîte I, Convocations.
} 
un président et, ensuite, parce qu'il y eut trop peu de représentants des É tats acceptant d'en faire partie...

Ce sont des personnalités de haut rang qui travaillent pour la $L$ ondon International A ssembly, au sein de laquelle les B elges occupent une place importante. Benjamin Ferencz, décrivant les membres de l'assemblée, explique en effet : " I ts members [... ] included such eminent personalities as $D r$. B enes of Czechoslovakia, $P$ rofesseur René Cassin of $F$ rance, the $L$ egal adviser to General de Gaulle, and the jurist Dr. M arcel de B aer of B elgium, who served as Chairman. $\gg 29$ Marcel de Baer est en réalité président de la commission I, chargée entre autres du dossier «the P unishment of War Criminals». II occupe donc une place centrale au niveau international pour l'étude de cette question, puisqu'il va également être nommé par le gouvernement comme représentant belge à la $\mathrm{U}$ nited $\mathrm{N}$ ation Commission for the I nvestigation of $\mathrm{W}$ ar Crimes30.

Henri Rolin est également présent au sein de l'assemblée, ce qui illustre un aspect moins connu de sa longue carrière politique ${ }^{31}$. II est en effet élu, en novembre 1942, «D eputy P resident » et préside à plusieurs reprises des séances de l'A ssemblée au cours desquelles de Baer fait notamment rapport del'évolution du travail de la Commission I concernant l'arrestation et les procès des criminels de guerre.

Dans le groupe belge de la $L$ ondon I nternational A ssembly, on retrouve des figures bien connues de l'establishment belge en exil à L ondres. IIs appartiennent principalement au monde politique, juridique, syndical et de la presse: J ef R ens, syndicaliste, homme politique socialiste et secrétaire général de la CEPAG 32 ; J ulius Hoste, directeur du journal H et L aatste

\footnotetext{
${ }^{29}$ B. B. Ferencz, A n International Criminal Court... , op. cit. , p. 56.

${ }^{30}$ A GR, P rocès-verbaux des séances du Conseil des Ministres, 13.11.1942.

31 Henri Albéric Rolin : «Homme politique socialiste (Gand 1891-Paris 1973). Docteur en droit et spécialiste du droit des gens, il enseigna à l'U L B. II siégea comme sénateur coopté et présida le Sénat de 1947 à 1949. À Londres, durant l'Occupation, il fut sous-secrétaire d'É tat à la Défense nationale (1942). II fut ministre de la J ustice durant quelques jours (1946). II siégea dans diverses assemblées internationales (SDN, ONU, Conseil de l'Europe, Cour permanente d'Arbitrage), où il œuvra au service de la paix. », dans H. H asquin (éd.), Dictionnaire d'H istoire de B elgique: Les hommes, les institutions, les faits, le Congo belge et le Ruanda-U rundi, Namur, 2000, p. 562. Voir surtout R. Devleeshouwer, H enri Rolin 1891-1973, U nevoix singulière, une voix solitaire, Bruxelles, 1994.

$32 \mathrm{La}$ Commission pour l'É tude des P roblèmes d'A près-Guerre (CE PAG) est une institution créée au début de l'année 1941 par le gouvernement en exil à Londres pour préparer l'après-guerre. Elle est placée sous la tutelle du premier ministre $\mathrm{H}$ ubert $\mathrm{P}$ ierlot et regroupe un nombre important de personnalités du monde intellectuel de la société belge en exil. Voir D. de B ellefroid, «The Commission pour l'É tude des Problèmes d'A près-guerre», dans M. Conway - J. Gotovitch (éds.), E urope in Exile: E uropean Exile Communities in B ritain 1940-45, Oxford, 2001, pp. 121-133.
} 
$N$ ieuws, sous-secrétaire d'É tat du gouvernement belge en exil à $L$ ondres ; L ouis de B rouckère, sénateur socialiste et professeur à I'UL B ; J ean Leroy, membre de la Sûreté de l'État; Marc Schreiber, chef de cabinet du ministre de I'I nformation; Arthur Wauters, ministre de l'I nformation ; I sabelle Blume, députée socialiste, et Charles Tschoffen, chef de cabinet du ministre de la J ustice.

Les travaux de la Commission I débutent de manière significative en septembre 1942 et vont déboucher sur le vote par l'Assemblée de résolutions importantes en matière de crimes de guerre.

A près six mois de travaux, Marcel de Baer, président de la Commission I, présente à l'A ssemblée, le 28 septembre 1942, un rapport sur les procès contre les criminels de guerre dans lequel il explique que le jugement des criminels de guerre est l'un des problèmes les plus complexes du droit international vu l'absence de loi positive en la matière. II s'appuie sur les erreurs commises après la Première Guerre mondiale et propose, pour ne pas répéter le fiasco, différentes mesures à prendre33.

Pour que la répression soit efficace, il faut qu'elle soit rapide et qu'elle ait lieu immédiatement après la signature de l'armistice: les procès ne doivent pas avoir lieu trois ans après la fin de la guerre, comme ce fut le cas lors du premier conflit mondial. II faut également que le principe du jugement des criminels de guerre soit inclus dans le traité d'armistice et non dans le traité de paix. $\mathrm{D}$ 'autre part, les procès ne peuvent être gérés par les Allemands eux-mêmes, comme ce fut aussi le cas lors des procès de L eipzig, mais sous l'égide des Nations U nies. II demande que soit établi le plus rapidement possible un protocole d'accord entre les forces alliées qui définisse les actes devant être poursuivis comme crimes de guerre, la loi qui leur sera applicable, ainsi que la mise en place d'un dispositif de recherche, sorte de police interalliée qui soit opérationnelle dès le cessez-lefeu. II insiste sur la nécessité d'une codification du droit pénal international, sur l'instauration d'une Cour pénale internationale, d'une révision des traités d'extradition et du droit d'asile dans les pays neutres.

La notion de crimes de guerre est difficile à circonscrire par les juristes et les définitions qui lui seront données pendant et même après la guerre seront diverses. Ainsi, de Baer précise ce que la Commission entend par crimes de guerre: "By "war crimes", we mean only the crimes which are

${ }^{33} \mathrm{AGR}$, procès-verbaux des séances du Conseil des Ministres. 
sufficiently hei nous and important to make them the concern of humanity » et les différencie de la trahison, dont les crimes visent une nation en particulier. $\mathrm{N}$ 'ayant pas de caractère international, ces crimes tombent sous le coup de la loi du pays concerné et relèvent des seuls tribunaux nationaux. Lors de la réunion du 23 août 1944 de la Commission I consacrée à la liquidation de la guerre, une autre définition sera donnée aux crimes de guerre: «W ar crimes are any grave outrages violating the general principles of criminal law as recognised by civilised nations and committed in war-time or connected with the preparation, the waging or the prosecution of war, or perpetrated with a vi ew to preventing the restoration of peace. » $34 \mathrm{E} n$ vertu de cette définition, la Commission met en évidence quatre types de crimes de guerre: la préparation d'une guerre d'agression déjà énoncée le 26 septembre 1942 par le professeur R ené Cassin, la violation des lois et coutumes de la guerre dont une liste substantielle est établie en décembre 1942, I'extermination d'une race, nation ou parti politique, et les crimes commis après la fin des hostilités pour entraver la paix 35 .

Plus tard, le Statut du Tribunal militaire international de Nuremberg du 8 août 1945 désignera le crime d'agression à l'article 6a sous l'appellation de «crime contre la paix »; il en fera un crime autonome, considéré comme le crime international suprême. Quant aux crimes de guerre, le Statut les définit comme des «violations aux lois et coutumes de la guerre. Ces violations comprennent les assassinats, les mauvais traitements, la déportation des populations civiles dans les territoires occupés; I'assassinat ou les mauvais traitements des prisonniers de guerre; l'exécution des otages; le pillage des biens publics ou privés; la destruction des villes; la dévastation non justifiée des objectifs militaires. »36 S'agissant des crimes liés à l'extermination, le Statut de Nuremberg l'incorpore dans la définition du crime contre l'humanité et lie les crimes à caractère racial ou politique aux persécutions; la volonté d'éliminer une nation n'y est pas mentionnée. Cette incrimination, considérée par certains comme contraire au principe de la non-rétroactivité de lois, parce qu'elle crée des notions nouvelles, contient les actes suivants: " L'assassi-

\footnotetext{
34 I bid.

${ }^{35}$ Cité dans B. B. F erencz, A n I nternational Criminal Court... , op. cit. , p. 100.

$36 \mathrm{G}$. H ermann, P rocès des grands criminels de guerre devant le tribunal mili taire international : $\mathrm{N}$ uremberg, 14 novembre 1945 - 1 ${ }^{\text {er }}$ octobre 1946, Nuremberg, International Military Tribunal, 1947, p. 266. Voir aussi R. Gutman - D. Rieff (éds.), Crimes de guerre. Ce que nous devons savoir, Paris, 2002, pp. 144-146.
} 
nat, l'extermination, la réduction en esclavage, la déportation, ou tout autre acte inhumain commis contre toutes populations civiles, avant ou pendant la guerre, ou bien les persécutions pour des motifs politiques, raciaux ou religieux, ou lorsque ces actes ou persécutions, qu'ils aient constitué ou non une violation du droit interne du pays où ils ont été perpétrés, ont été commis à la suite de tout crime rentrant dans la compétence du tribunal, ou en liaison avec ce crime. $» 37$

La loi belge du 20 juin 1947 relative à la compétence des juridictions militaires en matière de crimes de guerre, quant à elle, ne donne aucune définition du crime de guerre. Elle précise uniquement que les juridictions militaires connaîtront «des infractions tombant sous le coup de la loi belge et commises en violation des lois et coutumes de la guerre $» 38$. Si on se reporte aux travaux parlementaires qui précèdent la loi, en particulier au rapport fait par Marc Somerhausen, rapporteur de la Commission de la J ustice de la Chambre, la notion de crimes de guerre doit s'entendre au sens large. En dessous du sous-titre « définition des crimes de guerre », le rapporteur énonce en effet les qualifications formulées par le Statut du Tribunal militaire international de Nuremberg en englobant tout ensemble l'article $6 b$, qui se rapporte aux crimes de guerre, et l'article $6 c$, qui décrit les crimes contre l'humanité. II explique que « les faits énumé rés ci-dessus constituent des violations du droit interne de la Belgique, dans tous les cas où ils n'étaient pas autorisés par les lois et coutumes de la guerre »39. R apporteur de la Commission J ustice pour le Sénat, H enri $R$ olin tient des propos plus restrictifs : par manque d'audace et préoccupé essentiellement par le fait de ne pas porter atteinte au sacro-saint principe de la non-rétroactivité des lois, les parlementaires belges ont donc imposé, pour qu'il y ait crime de guerre, la double condition d'une atteinte à la loi pénale belge et aux lois et coutumes de la guerre. R olin le justifie en ces termes : «N ullum crimen, nulla poena sine lege. Le Tribunal international de Nuremberg a pu déroger à cette règle pour le châtiment d'actes qui avaient gravement offensé la conscience juridique universelle. II eût été peu raisonnable, peu opportun aussi, d'imposer à une juridiction nationale pareille responsabilité. La simple application de notre Code pénal

\footnotetext{
37 | bid.

$38 \mathrm{P}$ asinomie, 1947, p. 455.

39 D ocuments parlementaires, R apport de la Commission de la J ustice de la Chambre, session 1946$1947, n^{\circ} 311$, p. 3.
} 
suffira au surplus à assurer la répression des crimes dont nos ressortissants furent trop souvent les malheureuses victimes. $\gg 40$

Dans les procès qui auront lieu entre 1948 et 1951 à charge des criminels de guerre allemands devant les juridictions militaires belges, c'est la conception issue des propos de R olin qui prévaudra de façon constante. $\mathrm{H}$ ormis le premier procès, qui eut lieu au mois d'août 1948 devant le Conseil de Guerre de Charleroi, aucun des jugements suivants ne fera mention dans ses attendus de la notion de crime de guerre au sens large, les mots « déportation », repris dans I'article $6 b$ du Statut de N uremberg relatif au crime de guerre, ou celui de «persécution», repris à l'article 6c pour le crime contre l'humanité, n'ont plus leur place dans les procès. Ils sont censés être contenus dans celle "d'arrestation et détention arbitraire », prévue aux articles 434 et suivants du Code pénal belge.

Une question très importante est celle de la loi à appliquer et de la juridiction compétente en la matière : doivent-elles être nationales ou internationales? Quelle est la position de la L ondon I nternational A ssembly? L ors de la même séance du 28 septembre 1942, le président de la Commission I , Marcel de B aer, explique à I'A ssemblée que la Commission, excepté le délégué polonais, défend l'idée d'un code de droit pénal international. Selon lui, ce ne devrait pas être aussi compliqué qu'il n'y paraît puisque, hormis la coutume internationale, il suffirait de se fonder sur des textes comme les Conventions de La Haye et de Genève, qui devraient à l'évidence être réadaptées et complétées, notamment en fixant des peines pour chaque crime (ces conventions établissent en effet des infractions aux lois de la guerre, mais ne prévoient pas de sanction à leur violation). Le fait que de nombreux gouvernements soient réunis à Londres devrait de surcroît faciliter les choses.

Dans son argumentaire, le président de la commission considère que I'application de la loi nationale à ce type de crime est une solution non satisfaisante, parce qu'elle porterait atteinte au principe d'uniformité. L es criminels de guerre seraient en effet jugés sel on des codes différents et des procédures différentes, en particulier dans le cas d'actes perpétrés sur plusieurs territoires. II évoque aussi le cas de crimes commis en territoire ennemi et l'hypothèse inconcevable que ces faits soient jugés selon le code allemand en vigueur à la fin de la guerre.

40 D ocuments parlementaires, Rapport de la Commission de la J ustice du Sénat, session 1946-1947, $n^{\circ} 113$, p. 3. 
Quant aux juridictions compétentes, la question soulève au sein de la Commission des divergences de vue qui seront définitivement résolues lors de la séance du 9 juin 194341. Certains défendent l'idée que seule une Cour internationale de standing exceptionnel est à même de juger de tels crimes, qui concernent non pas un pays en particulier mais l'humanité entière. D'autres sont d'avis qu'il faut privilégier la compétence des tribunaux nationaux en conformité avec la règle générale selon laquelle l'inculpé doit être jugé par la juridiction du lieu ou le fait a été commis, moyennant certaines exceptions dont il sera question plus loin. C'est cette deuxième conception qui sera finalement retenue. Dans son rapport du 9 juin, la Commission I recommande le principe du jugement des crimes de guerre par les juridictions nationales (civiles, militaires ou mixtes) en conformité avec les lois en vigueur dans le pays concerné. Elle suggère donc aux É tats de prendre des mesures législatives nécessaires pour étendre la compétence des juridictions aux crimes commis à l'étranger contre leurs nationaux. C'est la raison pour laquelle la B elgique édictera, le 5 août 1943, un arrêtéloi « conférant compétence exceptionnelle aux juridictions belges concernant certains crimes ou délits commis en temps de guerre hors du territoire national », en rapport direct avec cette résolution.

Mais la Commission favorise l'établissement d'une Cour internationale dans quatre cas résiduels :

- les crimes pour lesquels aucun tribunal des Nations U nies n'a compétence, tels que les crimes commis en Allemagne contre les J uifs, les apatrides et les Alliés nationaux ;

- les cas où les tribunaux des Nations Unies ont compétence, mais décident de ne pas engager de procès vu le risque de troubles ou la trop grande difficultéà réunir les preuves;

- les crimes qui ont été commis dans différents pays ou contre des nationaux de différents pays;

- les crimes commis par les chefs d'É tat.

L a Commission réaffirme aussi le principe, déjà posé par l'I nternational Commission for $P$ enal R econstruction and D evelopment, selon lequel I'ordre d'un supérieur n'est pas une excuse et recommande de prendre toutes les mesures préparatoires, nationales ou internationales, pour que les poursuites puissent être entreprises dès la fin du conflit.

${ }^{41}$ A GR , ibid., boîte II , Commission I . 
Quelle influence la London International A ssembly a-t-elle eue sur la préparation par les $\mathrm{N}$ ations $\mathrm{U}$ nies des mesures à prendre pour le jugement des criminels de guerre? C'est elle qui, par l'intermédiaire d'un de ses membres, Lord Maugham, initie début septembre 1942 un débat important à la Chambre des $L$ ords sur cette question, débat qui est à l'origine de la déclaration commune du 7 octobre 1942 (citée supra) du président R oosevelt et d'Anthony $E$ den, ministre britannique des Affaires étrangères, par laquelle ils affirment leur volonté de ne pas répéter les erreurs de 1918 en promettant un jugement rapide des criminels de guerre dont le principe sera contenu non pas dans le traité de paix, mais dans l'accord d'Armistice. Ils annoncent aussi I'absence d'immunité pour les hauts responsables et la création d'une $\mathrm{F}$ act $\mathrm{F}$ inding Commissi on ${ }^{42}$. L e travail de la L ondon International A ssembly a donc exercé une influence sur les prises de position des chefs d'É tat, mais il a aussi balisé celui de la U nited $\mathrm{Na}$ tions W ar Crimes Commission (U N W CC), au sein de laquelle ont d'ailleurs été nommés par leurs gouvernements respectifs six membres de l'A ssembly (dont Marcel de Baer). Son projet de Convention sur la création d'une Cour pénale internationale préparé en 1943 a aussi marqué de son empreinte la rédaction des Statuts de N uremberg et de T okyo43.

$\mathrm{Par}$ conséquent, ce n'est pas, comme l'explique I'historien A rieh K ochavi, la seule U nited $N$ ati ons W ar Crimes Commission (UN W CC) qui est à l'origine de l'élaboration de certaines notions essentielles du Statut de Nuremberg, mais aussi un organisme comme la L ondon International A ssembly qui, dès la fin 1942, "was beginning to anticipate many of the doctrines which were later to form the basis for future war crimes trials. They were also able to study the arguments for an International Criminal Court which the internati onal community had pushed back into the closet. »44

\section{La préparation de la répression au niveau belge}

Avant d'examiner la politique adoptée par les autorités belges à Londres en matière de répression et la manière dont la B elgique s'inséra

\footnotetext{
42 B. B. Ferencz, A n International Criminal Court... op. cit., p. 58. AGR, ibid., boîte II, Commission II, Report on the P uni shment of W ar Criminals, CE GES, B elgi um, 1942, vol. III, n 10.

43 D raft Convention for the Creation of an International Criminal Court, dans B. B. F erencz, A n I nternational Criminal Court... op. cit., Document Ten, pp. 399-413. Voir aussi : T. L. H. McCormack - G. J . Simpson, The L aw of W ar Crimes..., op. cit., p. 56.

${ }^{44}$ B. B. Ferencz, A n International Criminal Court..., p. 58.
} 
alors dans la dynamique internationale, une remarque générale sur les archives s'impose.

L es archives en matière de répression des crimes de guerre en B elgique ne sont foisonnantes que dans leur dimension strictement législative et judiciaire, à savoir quand elles procèdent de la loi du 20 juin 1947, de sa préparation et de ses commentaires, ainsi que des procès proprement dits qui ont pu avoir lieu après sa promulgation longuement attendue. La période qui précède, celle de l'exil à Londres, est relativement pauvre en documents sur le sujet, par absence de sources et parce que la préoccupation dominante est celle "du châtiment des traîtres".

Le constat de carence en matière de sources concerne la double problématique des crimes de guerre et des persécutions commises contre les J uifs. S'il y a peu d'archives disponibles traitant de la question de la répression belge des crimes de guerre, il n'y en a pratiquement aucune qui, dans le cadre de ce sujet, documente la sanction judiciaire du génocide des J uifs (la notion de génocide ne sera consacrée légalement que le 9 décembre 1948 par la Convention pour la prévention et la répression du crime de génocide).

Par ailleurs, il faut souligner la présence des rapports de la $L$ ondon International A ssembly dans de multiples fonds d'archives, ce qui démontre l'importance accordée à l'époque au travail réalisé par cet organisme. Ils se trouvent à la fois dans les archives du cabinet du premier ministre à L ondres, dans celles du ministère des Affaires étrangères, dans les archives du H aut-Commissariat à la Sûreté de l'É tat et dans celles de la Commission des Crimes de Guerre.

Cependant, à partir des archives consultées, il est possible d'énoncer un certain nombre d'observations sur les intervenants bel ges et les démarches qu'ils ont entreprises, et d'en tirer ensuite certaines conclusions.

\section{Les intervenants belges}

L es intervenants belges qui prennent part au règlement par voie judiciaire des crimes commis par les Allemands sont peu nombreux. Quatre personnes y travaillent à la fois au niveau international et national.

L e personnage clef est incontestablement Marcel de B aer, qui s'investit à la fois dans I'I nternational Commission for P enal R econstruction and Development, la L ondon International A ssembly et dans la $U$ nited $\mathrm{N}$ ations W ar Crimes Commission. Au niveau national, il occupe le poste de prési- 
dent de la Cour militaire et de vice-président du Conseil juridique45. II y a aussi H enri Rolin, présent au sein de la London International A ssembly, qui exerce la fonction de sous-secrétaire d'É tat à la Défense nationale du 11 février 1942 au 2 octobre 1942 et qui siège au Conseil juridique, où il intervient de manière significative au sujet des modifications à apporter aux articles du Code pénal relatifs à la répression de la collaboration ${ }^{46}$. On retrouve enfin Charles Tschoffen, chef de cabinet du ministre de la J ustice et membre également du Conseil juridique, et Georges K aeckenbeeck, jurisconsulte au ministère des Affaires étrangères et président, entre 1942 et 1943, du Comité interallié pour la R épression des Crimes de Guerre.

A u niveau strictement national, les deux intervenants principaux dans le domaine de la répression sont le ministre de la J ustice, Antoine Delfosse, et le haut-commissaire à la Sûreté de l'État, Walter Ganshof van der Meersch ${ }^{47}$. Participe également au débat sur les crimes de guerre, $\mathrm{H}$ enri (E ugène) R olin, homonyme de l'homme politique. Président de la Cour de Cassation, il démissionne en décembre 1940 et s'exile aux É tats-U nis, où il rédige plusieurs études de droit constitutionnel et de droit international et publie de nombreux articles dans B elgium, importante publication éditée à N ew Y ork ${ }^{48}$. L e 31 mai 1943, il écrit une note à l'attention de la CEPAG, dans laquelle il réagit suite à un article publié dans le $\mathrm{N}$ ew $Y$ ork Times par Charles W arren, A ssistant A ttorney General honoraire aux É tats-Unis ${ }^{49}$. Dans la partie réservée aux jugements par des tribunaux militaires internationaux, il se prononce résolument contre l'instauration de ceux-ci : «II semble que le caractère international des juridictions dont la création est préconisée exerce sur l'esprit de certains juristes une séduc-

\footnotetext{
45 Le Conseil juridique a pour mission de préparer des projets d'arrêtés-lois ou d'arrêtés et de donner un avis sur des questions dont il est saisi par le gouvernement. Voir H. Fayat, L égislation belge en exil : aperçu de l'action législative et exécutive du Gouvernement belge en exil, 16 mai 1940 - 8 septembre 1944, B ruxelles, 1994, p. 12.

${ }^{46}$ Sur l'activité d'H enri R olin pendant l'exil à Londres, voir R. Devleeshouwer, H enri Rolin : 18911973, une voix singulière, une voix solitaire, B ruxelles, 1994, pp. 279-321.

47 Docteur en droit de l'ULB, Walter Ganshof van der Meersch est nommé en avril 1940 auditeur général auprès de la Cour militaire. R ésistant, il arrive en juin 1943 à L ondres, où il est nommé hautcommissaire à la Sûreté de l'État. A près la guerre, il continue à occuper les fonctions d'auditeur général jusqu'en 1947, date à laquelle il devient avocat général, puis procureur général à la Cour de Cassation.

48 Certains numéros de B elgium sont consultables au CE GES. Sur Henri Eugène R olin, voir W. J . Ganshof van der M eersch, N otice sur H enri R olin, B ruxelles, 1978.

${ }^{49} \mathrm{AMAE}$, dossier 11767, Commission bel ge pour l'étude des problèmes d'après-guerre. Châtiment des coupables.
} 
tion puissante, mais peu justifiée. L e grand défaut de pareilles juridictions sera d'être improvisées, de manquer de tradition et de cohésion. A u fond, ce sont des tribunaux extraordinaires, dont I'article 94 de la Constitution interdit la création. » Selon H enri R olin, la loi pénale belge couvre toutes les situations: "L es actes de violence, interdits par les "lois de la guerre" et par les lois d'humanité, tombent sous l'application des lois pénales de droit commun, antérieures à l'invasion de 1940 et toujours en vigueur en Belgique. La prise d'otages, les arrestations arbitraires, les exécutions avec ou sans jugement, les confiscations de toutes espèces [... ] tombent sous le coup de quantité de dispositions, contenues notamment dans les titres VIII et IX du Livre II du Code pénal. » Quant aux tribunaux compétents, il suggère d'étendre celle des Conseils de Guerre pour juger de ce type de crimes et délits.

$L$ a position défendue par H enri $E$ ugène R olin, président de la Cour de Cassation, est exactement celle qui sera soutenue par les parlementaires de 1946-1947, auteurs de la loi relative aux crimes de guerre. On peut cependant s'étonner de cette position strictement légaliste pour quelqu'un qui fut sensible aux persécutions antijuives, qui apporta son aide aux étudiants juifs venus d'Autriche et d'Allemagne et dont l'épouse, d'origine juive, perdit la plupart des membres de sa famille dans les camps dela mort50.

L a prise d'otage, les persécutions, la déportation, et la torture ne sont pas incriminées en tant que telles dans le code pénal. Ces carences juridiques ne sont étrangement pas décelées ou, si elles l'ont été, la volonté d'y remédier est pour le moins absente de tous les dossiers. À l'exception d'une lettre d'H ubert Pierlot et d'une note de Charles Tschoffen, aucune archive consultée ne fait mention d'une préoccupation ou d'un débat parmi les autorités belges sur cette question précise.

\section{Les démarches entreprises par ces différents intervenants}

Deux périodes peuvent être distinguées sel on que l'on se situe avant ou après le 1er novembre 1943, date de la Déclaration de Moscou, qui fixe définitivement et de manière officielle le principe de la compétence nationale pour le jugement des criminels de guerre avec cependant une compé-

50 W. J . Ganshof van der M eersch, ibid., pp. 32-33. 
tence internationale résiduelle pour les crimes qui ne sont pas localisés géographiquement.

Durant la période qui précède cette décision, les choses bougent. Le premier ministre et le ministre de la J ustice semblent décidés à faire tout ce qui est nécessaire pour rendre l'arsenal juridique belge le plus efficace possible. Mais, après novembre 1943, plus rien n'est entrepris et les choses restent en l'état. Un mot reflète l'attitude des autorités belges en matière de crimes de guerre : I'attentisme.

L e gouvernement prend une première décision en publiant un arrêtéIoi, daté du 10 janvier 1941, qui prévoit que toutes les mesures prises par l'occupant seront tenues pour abrogées de plein droit au fur et à mesure de la libération du pays, de même que les mesures de dépossession prises par l'occupant seront tenues pour nulles et non avenues 51 . Ceci s'applique par conséquent aux mesures antijuives prises par l'administration allemande, ce que le gouvernement ne manque pas d'évoquer à différentes reprises. L e premier ministre aborde toujours la question des persécutions de manière plus ou moins semblable: dans ses différentes interventions, il rappelle les principes de liberté et d'égalité constitutionnelles et évoque la victoire des Alliés comme unique solution pour rétablir ces principes, aucune autre mesure concrète n'étant avancée. II n'est toutefois pas le seul parmi les gouvernants alliés à tenir ce discours: le Commissaire national aux Affaires étrangères du Comité national français, Maurice D ejean, ne dit pas autre chose dans son message d'avril 1942 publié dans le J ewish Bulletin 52 .

A u niveau international, la Belgique s'engage en janvier 1942, lors de la Conférence de Saint-J ames, aux côtés des autres gouvernements en exil à $L$ ondres pour dénoncer les violations des lois de la guerre commises par I'Allemagne et menacer celle-ci de sanctions judiciaires après la guerre53. L e gouvernement semble imprégné de cette volonté de ne pas réitérer les erreurs du passé et de prendre toutes les mesures utiles pour se mettre en conformité avec les décisions prises à l'échelon international. Le 14 novembre 1942, H ubert P ierlot envoie au nom du gouvernement un rapport au comité Gilles, dans lequel il fait état de la situation générale à L ondres

\footnotetext{
51 L es deux arrêtés-lois sont de la même date : A GR, PV du conseil des ministres, 14.01.1941.

${ }_{52} \mathrm{AGR}$, Cabinet du premier ministre à L ondres, dossier 313.

53 Voir $P$ unishment of $W$ ar Crimes. The Inter-Allied Declaration, déclaration du premier ministre H ubert Pierlot, dans AGR, Cabinet du P remier ministre à L ondres, dossier 513.
} 
et aborde notamment la question des crimes de guerre ${ }^{54}$. II s'adresse à Ganshof van der Meersch, membre du comité, auquel il fait part des décisions prises lors de la Conférence de Saint-J ames: "La Conférence des Nations Unies, qui a tenu plusieurs séances au Palais de Saint-J ames, a arrêté, au sujet des crimes de guerre, les termes d'une déclaration de principe dont vous avez eu connaissance, notamment par la radio. $D$ 'après ces règles, tous les criminels de guerre, sujets des pays ennemis ou traîtres appartenant à des nations alliées, seront punis selon les rigueurs du droit commun, respectivement par ceux des pays intéressés auxquels ils seront remis, soit en raison de leur nationalité, soit en raison du territoire sur lequel les infractions auront été perpétrées. Ce n'est point là une déclaration vaine destinée à rester lettre morte, comme celles qui ont été faites au sujet des crimes de la guerre 1914-1918. La résolution des Nations U nies prend, dès à présent, un caractère organique. Indépendamment de la Commission permanente chargée de mettre au point la législation internationale relative aux crimes de guerre, un autre organisme a été créé, sous le nom de " $\mathrm{F}$ act $\mathrm{F}$ inding Commi ssion" et qui a le caractère d'une véritable juridiction d'instruction, chargée de rechercher les criminels, de composer - autant qu'on le peut dès maintenant - leurs dossiers et de déterminer quelles sont les juridictions nationales devant lesquelles ils seront traduits. $\gg 55$ Le premier ministre y affirme le principe de l'application de la loi nationale et de la compétence juridictionnelle nationale aux criminels de guerre ennemis et aux collaborateurs belges, pour ensuite mentionner la mise au point future d'une législation internationale. Mais à ce stade, rien n'est encore très clair en la matière. Le plus intéressant est surtout ce qui suit : «II importe que le P euple belge sache que justice sera faite, dans le Pays et au-dehors, avec une inflexible rigueur. Le gouvernement belge fait, d'autre part, examiner quels sont les articles du Code pénal qu'il y aurait lieu de modifier pour les mettre en concordance avec ces projets. Les projets de textes nouveaux vous seront communiqués ultérieurement. » Dans ce rapport, le premier ministre ne fait pas de la communication publique; il s'adresse secrètement à celui qu'il nommera à la tête de la Sûreté de l'É tat dès son arrivée à $L$ ondres et qui sera I'homme de réfé-

\footnotetext{
54 L e comité Gilles est créé par le gouvernement bel ge de L ondres. I l lui sert d'antenne clandestine en Belgique. Voir T. Grosbois, P ierlot 1930-1950, B ruxelles, 2007, pp. 261-262.

55 Université libre de B ruxelles (ULB), Papiers Ganshof van der M eersch, 180 PP/080.016, Mémoires: comité Gilles.
} 
rence en matière de répression 56 . L 'intention de poursuivre est donc bien réelle dans un message où il est davantage question - une fois n'est pas coutume - de crimes de guerre commis par des étrangers que de poursuites contre les nationaux. Mais ce n'est pas seulement de détermination à poursuivre qu'il est fait mention ici ; c'est aussi du problème de la modification du Code pénal dont il s'agit, modifications qui devraient assurer la conformité de la législation belge avec les projets internationaux. Or, dans les deux années qui suivront, un seul arrêté-loi lié aux crimes de guerre sera pris par le gouvernement en exil et aucun article du Code pénal ne sera modifié en vue de préparer au mieux le jugement des criminels de guerre allemands. T out restera en quel que sorte en attente.

L e volontarisme en matière de jugement des crimes de guerre est aussi particulièrement présent dans les discours du ministre de la J ustice Antoine $D$ elfosse. Ses principales interventions sur la question s'échelonnent le long de l'année 1943 ; en 1944, il interviendra uniquement à propos des mesures prises à l'encontre des collaborateurs. Les propos qu'il tint au sujet du traitement de l'Allemagne après la guerre sont durs et parfois teintés d'appels à la vengeance. Dans une allocution prononcée à la radio le 14 janvier, il déclare: "Le traité de paix tranchera définitivement le sort de l'Allemagne et la mettra pour jamais hors d'état de nuire. Mais il appartiendra aux gouvernements alliés de tirer vengeance - une vengeance légitime et conforme à la morale des pays civilisés - de tous les criminels qui ont violé les lois de la guerre. »57 L e jour précédent, dans un discours prononcé au luncheon anglo-américain où H ubert Pierlot est aussi présent, il rappelle les déclarations de R oosevelt et Churchill sur le jugement des criminels de guerre et ne s'embarrasse d'aucune précaution particulière pour s'exprimer sur l'ensemble du peuple allemand: "Permettez-moi de vous apporter le témoignage d'un pays qui n'a jamais voulu de mal à personne et qui sait que les Allemands ne sont bons que quand ils sont battus et rendus inoffensifs [... ]. L es Allemands ne seront supportables pour leurs voisins que quand ils auront accepté, dans le concert des nations, de vivre comme les autres. $\gg 58$

\footnotetext{
56 Voir P. A ron - J. Gotovitch (éds.), Dictionnai re de la Seconde Guerre mondiale en B elgi que, Bruxelles, 2008, pp. 380-386.

${ }^{57}$ CE GE S, I nbel, 1003, 14.01.1943.

${ }_{58}$ CE GE S, Archives Marc Schreiber, dossier n 3, A nglo-A merican L uncheon, 13.01.1943.
} 
E n septembre de la même année, il s'exprime de façon similaire devant le Commi ttee for Study and Lecture en présence de membres des gouverne ments anglais, polonais, tchécoslovaque, français et belge: "Comment traiter l'Allemagne après la guerre? L es avis sont partagés : les uns disent il suffit de chasser le nazisme; le peuple allemand n'est pas responsable. J e ne suis pas d'accord. J uridiquement et moralement, le nazisme est sorti du peuple allemand lui-même et se confond avec lui [... ] N'oublions pas non plus que le châtiment des crimes de guerre sera nécessaire pour montrer au peuple allemand que non seulement la guerre est une industrie qui ne profite pas à l'É tat, mais encore que les individus ne la déclenchent pas impunément. »59 Ce châtiment, il le considère sous un double aspect : militaire et judiciaire, vengeance et justice. Dans une allocution prononcée à la radio le 9 octobre, il explique: "Les bombardements de l'Allemagne notamment, outre qu'ils sont un des moyens efficaces de finir la guerre, rendent aux agresseurs la monnaie de leur pièce, parce qu'ils punissent durement cette population qui applaudissait sans scrupule à la destruction de Rotterdam, de Tournai ou de Coventry. Mais cela ne suffit pas pour rétablir la justice violée. II faut punir individuellement ceux qui ont conduit la guerre avec des méthodes de barbarie que la civilisation primitive aurait elle-même répudiées. Cette idée de sanction contre les criminels de guerre est inséparable de la victoire; elle sera une de ses premières conséquences. On peut dire qu'elle est une des conditions de son efficacité. $\gg 60$ Le 10 novembre, il accorde une interview aux représentants de la U nited P ress of A merica portant sur la conférence de Moscou et déclare entre autres: " Comme vous le rappelez, les B elges ont connu deux fois en une vie d'homme la même abominable invasion, les mêmes atrocités, les mêmes massacres d'otages, les mêmes brutales déportations. Déjà, après 1914, notre poète V erhaeren avait montré le cri de haine que poussait un peuple tout entier injustement frappé. Cette haine et un légitime désir de vengeance sont aujourd'hui plus intenses encore qu'autrefois. [... Les B elges ne supportent pas la pensée qu'une fois encore les coupables pourraient échapper à un juste châtiment. La Conférence de M oscou vient de leur donner des assurances formelles. » 61

\footnotetext{
${ }_{59}$ CE GE S, I nbel, 1003, 28.09.1943.

60 I bid., 9.10.1943.

61 I bid., 10.11.1943.
} 
Dans ces différentes interventions, le ministre de la J ustice prend soin d'utiliser le mot «Allemands », mais il lui arrive aussi d'opter pour celui de «boches », renvoyant du même coup au contexte de la Première Guerre mondiale. Ceci lui vaudra une «correction » de la part de Ganshof van der Meersch qui, sollicité par D elfosse au sujet de la rédaction d'une allocution à prononcer à la radio le 22 mars 1944, lui fait part de quelquesunes de ses réflexions: " $E n$ B elgique occupée, on ne se sert absolument pas du terme "boches". J e crois qu'il est peut-être dans ces conditions avisé de remplacer ce terme par "Allemands". „ 62

L es paroles prononcées par le ministre de la J ustice sont fermes et empreintes de détermination. Concrètement, toutefois, que propose-t-il pour punir les coupables? Peu de choses. Mais, pour le lecteur ou l'auditeur de l'époque, les discours donnent une impression différente: beaucoup d'éléments sont expliqués sur le travail international, celui de la $L$ ondon International A ssembly, de la Commission de Cambridge et du Comité interallié pour la R épression des Crimes de Guerre. II évoque aussi la création d'un parquet interallié, qui devrait régler le problème des extraditions. Sur le plan de la procédure, « il n'y a guère que sur le genre de tribunal qu'on peut discuter $\gg 63$. II se prononce pour la mise en place d'un tribunal international pour juger les grands criminels et pour un règlement local lorsqu'il s'agit de coupables de moindre envergure. L orsqu'il commente, dans une interview du 10 novembre 1943, les décisions prises à ce sujet à la Conférence de M oscou par les É tats-Unis, la Grande-B retagne et I'URSS, il sous-entend que ceux-ci n'ont finalement rien annoncé de très nouveau, contrairement à la Belgique et plus particulièrement à sa propre personne: «Le gouvernement belge a constaté avec une profonde satisfaction que ses vues étaient entièrement partagées par les Grandes Puissances alliées. Moi-même, il y a quelques semaines, j'ai dans un discours à la radio, préconisé un système de punition des coupables exactement semblable à celui qui fait l'objet de la déclaration de Moscou. »64

Quand il aborde les mesures nationales, il met d'abord en évidence les dispositions prises à l'égard des collaborateurs, contre lesquels le gouvernement à la fois renforce les sanctions pénales et prépare des mesures de procédure appropriées : les juridictions militaires sont en effet désignées

\footnotetext{
${ }^{62}$ A GR, H aut-Commissariat à la Sûreté de l'É tat, dossier $n^{\circ} 122$, Ministère de la J ustice. ${ }^{63}$ CE GE S, I nbel, 1003, 9.10.1943.

64 I bid., 10.11.1943.
} 
compétentes en matière de « crimes et délits contre la Sûreté extérieure de l'É tat », titre qui régit dans le Code pénal les infractions commises par les collaborateurs. Quant aux criminels de guerre allemands, tout porte à croire que les choses sont prises en main : la B elgique établit la liste des A llemands qui ont violé les lois internationales dont la $B$ elgique réclamera la remise au moment de l'armistice. II demande par la même occasion à la population de rassembler les informations qu'elle détient sur les crimes allemands, afin de faciliter la tâche de la justice le moment venu. Surtout, il affirme que le gouvernement a pris toutes les dispositions qui s'imposent en la matière: "Le gouvernement belge a d'ailleurs, depuis un certain temps, pris les mesures législatives qui s'imposent pour que nos tribunaux répressifs aient compétence pour juger tous les criminels allemands, qu'ils aient commis leurs forfaits sur des Belges ou des alliés, aussi bien en Belgique qu'à l'étranger. » 65 Le ministre de la J ustice fait ici référence à l'arrêté-loi du 5 août 1943, qui étend les compétences des juridictions aux crimes commis par des étrangers hors de la Belgique. Dans le rapport au Conseil qui précède le texte de loi, D elfosse explique : « Même si I'on constitue une juridiction répressive internationale, il apparaît bien que la ré pression envisagée restera en grande partie confiée aux juridictions des divers É tats intéressés. II nous incombe, dès lors, de rechercher si notre législation permet dans tous les cas l'exercice de l'action publique. »66 Cet arrêté-loi vise notamment les actes commis contre des Belges dans les camps allemands ou les faits commis en UR SS par la L égi on étrangère. La portée de cet arrêté-loi d'août 1943 sera d'ailleurs étendue par la loi du 20 juin 1947 (la loi étend en effet la compétence des juridictions à certaines infractions commises hors de B el gique par un étranger contre un ressortissant étranger résidant en B elgique au moment des hostilités).

Mais pour que les tribunaux aient effectivement compétence, il faut déterminer laquelle des juridictions civiles, militaires ou mixtes aura à connaître des crimes de guerre. Sur cet aspect précis et essentiel, rien n'est décidé. Aucune question posée, aucune réaction n'apparaît ni dans la presse, qui semble satisfaite du travail du ministre, ni au sein des organes qui entourent le gouvernement (conseil législatif, $\mathrm{H}$ aut-Commissariat à la Sûreté de l'É tat, CEPAG).

65 I bid.

${ }^{66} \mathrm{~A}$ GR , Cabinet du premier ministre à L ondres, dossier 513. 
Quant aux crimes commis par des étrangers en B elgique, la seule mesure prise est spécifiée par la circulaire du ministre de la J ustice $n^{\circ} 340$ sur l'internement des étrangers et de certains sujets belges que leurs relations avec l'ennemi rendent suspects67. Quant aux lacunes éventuelles que contiendrait le Code pénal, Antoine Delfosse semble considérer que celui-ci est suffisant, alors même qu'il dénonceles massacres d'otages et les brutales déportations: "Chaque pays a ses lois écrites ou coutumières; ceux qui ont violé les lois de la guerre, codifiées dans la Convention de La $\mathrm{H}$ aye, savaient à quoi ils s'exposaient suivant le Code pénal en vigueur là où ils se sont livrés à leurs excès. »68

De façon incompréhensible, parmi toutes les archives consultées jusqu'à présent, un seul document émanant d'une personnalité représentative parmi les autorités belges fait état d'un questionnement à la fois sur les juridictions compétentes et sur les lacunes juridiques à combler. II s'agit d'une note de Charles Tschoffen, chef de cabinet d'A ntoine D elfosse, mais qui date du 5 janvier 1943, période transitoire où rien n'est encore décidé au niveau international. L 'auteur la présente comme « une esquisse destinée à poser les problèmes et à suggérer des solutions. E lle n'a rien d'officiel $\gg 69$. Sans entrer trop avant dans les détails, relevons quelquesunes de ses propositions.

L e premier point concerne l'énumération des crimes de guerre. II divise ceux-ci en trois catégories: les actes ayant trait à la genèse de la guerre (déclaration de guerre, invasion en violation des traités existants), les actes commis pendant la guerre par l'ennemi (massacres de populations civiles) et, contrairement à l'opinion de Marcel de Baer, les actes de trahison des nationaux au profit del'ennemi.

S'agissant de la seconde catégorie, il suggère, parmi les actes commis sur ordre de l'autorité par des membres de l'armée ou services officiels ennemis, une liste de dix-sept infractions, qu'il précise être «à peu de

\footnotetext{
67 La circulaire n 340 du 21 août 1944 est prise en application de l'arrêtéloi du 12 octobre 1918. L'article 3 de la circulaire prévoit : «Seront internés et mis à ma disposition, les étrangers dont la présence est dangereuse pour le maintien de l'ordre public. Sont compris dans cette catégorie notamment les ressortissants allemands ou d'É tats alliés de l'Allemagne, âgés de plus de 16 ans accomplis qui se trouvaient sur le territoire du R oyaume au moment de sa libération ; les étrangers, membres ou auxiliaires de l'armée allemande ou d'une armée d'une puissance alliée à l'Allemagne, fonctionnaires ou employés qui ont été au service de l'ennemi, et, plus généralement, tous les étrangers qui ont notoirement favorisél'action de l'ennemi en B elgique. »

${ }^{68}$ CE GE S, I nbel, 1003, 9.10.1943.
}

${ }^{69} \mathrm{AMAE}$, dossier 11767, note du ministère de la J ustice du 5.01.1943. 
chose près celle de la section de la $L$ ondon A ssembly étudiant les crimes de guerre et présidée par $M$. de B aer ». Parmi celles-ci, on relève les mauvais traitements ou privation de liberté infligés à des innocents, la mise à mort d'otages, l'emprisonnement non judiciaire ou les tortures infligées à des civils ou à des prisonniers de guerre, y compris des travaux imposés gravement hors proportion avec les facultés physiques de l'individu, les déportations. Aucune mention n'est faite des persécutions raciales.

L e second point concerne la législation pénale à appliquer. Plus audacieux que son entourage, T schoffen défend l'application de la rétroactivité dans certaines conditions: "Sans doute objectera-t-on à la rédaction actuelle d'un Code pénal international le principe de la non-rétroactivité des lois pénales. Ce principe est évident lorsqu'il existe pour l'ensemble des crimes envisagés un "droit écrit", une codification des règles en vigueur. Lorsqu'il n'y a ni "droit écrit" ni codification, le principe est des plus discutables. Dans ce cas - celui qui nous occupe -, la rétroactivité peut être appliquée, à la condition que ce code à élaborer ne soit que l'application, soit de principes universellement admis, soit de conventions internationales. » Par conséquent, il propose que les actes de la première catégorie (actes ayant trait à la genèse de la guerre), pour lesquels il n'est prévu aucune sanction dans aucun code pénal, national ou international, et certains actes de la seconde catégorie non prévus par les lois nationales (déportation, tortures, mauvais traitements, massacres de civils) soient jugés suivant les principes de droit international et un code pénal à rédiger, le reste étant régi par la loi nationale de la victime.

$L$ a rétroactivité a donc ses limites. Tschoffen ne propose pas que les lois nationales soient modifiées ou complétées de façon à combler d'éventuelles lacunes juridiques.

I ci non plus, les archives ne font pas apparaître, parmi les autorités belges à L ondres, l'existence d'un débat de fond portant sur les lacunes du Code pénal. La question est pourtant clairement posée par Léon K ubowitzki, représentant du W orld J ewish Congress, à Marc Schreiber, chef de cabinet du ministre de l'I nformation, au sujet de l'incrimination spécifique des crimes commis contre les J uifs et de la position que le gouvernement belge compte adopter à cet égard : «I would be parti culary grateful to you for letting me know what the attitude is of the Belgian official circles towards the specifically anti J ewish crimes that are not covered by the general 
concepti on of $W$ ar Crimes. $\gg 70 L$ a réponse de Schreiber ne figure malheureusement pas dans les archives, mais elle n'est pas difficile à deviner: lorsque le gouvernement intervient pour dénoncer la politique raciale des Allemands, il répète inlassablement, d'une part, que les mesures antijuives seront tenues pour nulles et non avenues et menace, d'autre part, les B elges de sanctions pénales après la guerre pour l'aide qu'ils apporteraient à leur exécution. Le gouvernement se refuse à pratiquer une politique spécifique en faveur des J uifs dans quelque domaine que ce soit, parce que cela irait à l'encontre des principes constitutionnels qui interdisent toute forme de discrimination ${ }^{71}$. Cette attitude n'est pas différente de celle adoptée à l'échel on international, puisque Georges K aeckenbeeck, président du Comité interallié pour la Répression des Crimes de Guerre, explique dans un passage de son rapport portant sur les réclamations des organisations juives: "II a été expressément reconnu que la déclaration de Saint-J ames n'avait pas de caractère limitatif et que, si l'on n'avait pas év oqué explicitement les souffrances endurées par les J uifs, c'est parce qu'on estimait qu'une telle mention équivaudrait à une reconnaissance implicite des théories raciales que nous rejetons tous. »72

Un dernier intervenant, capital dans le domaine de la répression, est I'auditeur général Walter Ganshof van der Meersch. II arrive à L ondres en juin 1943 et est nommé le 4 août 1943 haut-commissaire à la Sûreté de l'É tat par les ministres réunis en Conseil. Le ministre de la J ustice le charge «d'examiner les projets d'arrêtés-lois ou d'arrêtés des ministres réunis en Conseil, concernant les modifications projetées de la compétence des juridictions à l'organisation judiciaire, ainsi qu'aux sanctions civiles et pénales nouvelles à prévoir pour les crimes et délits contre la sûreté intérieure et extérieure de l'E tat $\gg 73$. Que prépare-t-il en matière de crimes et délits commis par les étrangers? Au niveau des compétences des juridictions, rien. Pas plus qu'il ne met en garde le gouvernement des difficultés futures que risquent de rencontrer les juridictions en raison des lacunes contenues dans le Code pénal. Ses interventions sont en quelque sorte

\footnotetext{
70 CE GE S, Archives Marc Schreiber, dossier n 13, lettre du 27.08.1943.

71 Voir à ce sujet N. Wouters, «La persécution des J uifs devant ses juges», dans R. Van Doorslaer (éd.), op. cit., pp. 818-819.

72 AMAE, dossier 11767, R apport de M. K aeckenbeeck sur le travail du comité interallié pour la répression des crimes de guerre (avril 1942-avril 1943), 03.05.1943.

${ }^{73} \mathrm{~A}$ GR, Archives du H aut-Commissariat à la Sûreté de l'É tat, dossier $n^{\circ} 122$, lettre de Ganshof van der Meersch au ministre de la J ustice, 09.07.1943.
} 
périphériques par rapport aux crimes de guerre. Ceux-ci sont mentionnés, mais sans que les difficultés inhérentes à la matière soient abordées.

On peut s'en étonner, puisqu'en tant qu'auditeur général, il est chargé, dans le cadre de l'Office national belge pour l'É tude et la Documentation des Crimes de Guerre, d'établir et de communiquer ensuite à la U nited $\mathrm{N}$ ations $\mathrm{W}$ ar Crimes Commission les dossiers des crimes de guerre qui concernent la B elgique. II se plaint en juin 1944 auprès du ministre Delfosse du surcroît de travail occasionné à la Commission, ainsi que du manque de moyens qui la handicape, mais n'évoque pas le fait qu'à cette date aucun arrêtéloi n'est préparé pour désigner les tribunaux compé tents pour juger en Belgique, après la guerre, ces criminels de guerre ${ }^{74}$. Or un arrêté-loi est pris le 26 mai 1944, qui attribue compétence aux juridictions militaires pour connaître des crimes et délits commis par les collaborateurs belges, matière pourtant connexe aux crimes de guerre. II est par contre très vigilant et réactif lorsqu'il décèle d'éventuelles lacunes qui entraveraient la poursuite des Belges qui ont apporté une aide à l'ennemi. C'est ainsi qu'il écrit au ministre de la J ustice pour l'avertir que la Commission des Crimes de Guerre a été saisie de l'étude d'un projet de convention interalliée prévoyant la livraison de criminels de guerre entre pays alliés. Ce projet permettra à la Belgique de réclamer éventuellement la livraison, non seulement de criminels de guerre de diverses nationalités, mais encore des nationaux ou ex-nationaux belges qui se seraient enfuis en territoire allié et qui pourraient être poursuivis du chef d'infraction contre la sûreté extérieure de l'É tat. «Ceux-ci sont, en effet, manifestement compris dans les "other W ar Offenders" définis comme suit par l'art. II du projet: "All persons nationals and former nationals of the requesting state who are within their jurisdiction and are charged with or convicted of giving aid or comfort to the enemy or of an offence committed with the intent to further the cause of the enemy, or of an offence committed by means of the power or opportunity afforded by a state of war or armed hostilities, or by hostile occupation of territory of the requesting state." Par contre, rien n'a été fait jusqu'à présent, à ma connaissance, en ce qui concerne la livraison des personnes et notamment de nos nationaux qui devraient être poursuivis du chef d'une de ces infractions contre la Sûreté extérieure de l'État, et

${ }^{74} \mathrm{~A} \mathrm{GR}$, I bi d., lettre de Ganshof van der M eersch au ministre de la J ustice, 14.06.1944. 
qui se seraient enfuis en territoire ennemi. C'est là pour la Belgique une question qui me paraît d'intérêt essentiel. »75

II est tout aussi agissant lorsque les Alliés insistent auprès des autorités belges pour qu'elles prennent un arrêté-loi déclarant la justice militaire belge compétente au moment de la libération du territoire, afin de connaître d'un certain nombre d'infractions non prévues par la législation, commises par des sujets ennemis, aux clauses relatives à l'armistice. Dans le cas contraire, les Alliés menacent d'instaurer des M ilitary Courts 76 . L'auditeur prépare rapidement un projet d'arrêtéloi applicable uniquement aux étrangers, qu'il soumet au ministre de la J ustice et dans lequel sont érigés en infraction des faits qui, jusque là, n'étaient pas connus de la loi belge. II s'agit de: a) Incitation à la mutinerie des membres des troupes alliées. b) Corruption des membres des troupes alliées. c) Détention de carte d'identité, laissez-passer contrefait délivré par l'autorité militaire alliée. d) E mpêchement ou intervention dans les arrestations opérées par des officiers alliés. e) Action en vue de faciliter l'évasion des personnes détenues par les autorités alliées. f) Communication sans autorisation avec une personne détenue par les autorités militaires alliées. g) S'être fait passer pour un officier agissant sous l'autorité du $\mathrm{CIC}$. h) E nlever, détruire ou déplacer les textes des ordonnances et arrêtés, affichés sur ordre du $\mathrm{CI}$ C. i ) R efus d'obtempérer aux ordres donnés par les officiels alliés agissant sur ordre des autorités alliées. j) Se plaindre indûment ou faussement d'un officiel allié. k) Avoir, par distribution de journaux, tracts, etc., mené une propagande encourageant l'indiscipline auprès des armées alliées. L) A voir commis une infraction ou une tentative d'infraction aux ordonnances ou arrêtés du ClC. II s'agit toutefois là de faits qui ne constituent pas réellement des infractions au droit de la guerre : I'incitation à la désertion, par exemple, n'est pas un crime de guerre et n'avait donc pas été introduite dans la législation belge.

On constate néanmoins que, lorsqu'il s'agit de faits commis par les collaborateurs belges ou lorsqu'une pression suffisamment forte est exercée sur les autorités belges (en l'occurrence par les Alliés, qui semblent avoir fortement impressionné l'auditeur général, lequel a tout mis en œuvre pour les satisfaire), il n'y ait pas de difficultés majeures à légiférer, que

\footnotetext{
75 I bid., note de Ganshof van der Meersch au ministre de la J ustice, 28.08.1944.

${ }^{76} \mathrm{AGR}$, Cabinet du premier ministre à Londres, dossier $\mathrm{n}^{\circ} 765$, note de Ganshof van der Meersch au ministre de la J ustice, 07.04.1944.
} 
ce soit pour donner compétence aux juridictions militaires ou créer, malgré le principe de la non-rétroactivité des lois, une infraction nouvelle.

\section{L'attentisme du gouvernement de Londres}

Deux ans plus tard, lors d'une réunion le 20 septembre 1946 de la Commission internationale permanente pour l'É tude de la R épression des Crimes contre le Droit des Gens et des F aits commis dans l'intérêt de I'E nnemi, Ganshof van der M eersch prend la parole et fait part aux délégués des différents pays de la proposition faite à la Belgique par I'I nternational L aw Commission de faire juger par le tribunal international les principaux criminels de guerre allemands réclamés par la Belgique. Sans grand embarras, il explique aux membres de la Commission que: «Son avis fut demandé. II a été très favorable à cette proposition. Certains criminels sont impossibles à juger en Belgique, notamment le général von $\mathrm{F}$ alkenhausen, toute la collaboration administrative pouvant être évoquée, s'il devait être jugé en Belgique. »77 Déjà en avril de la même année, l'auditeur général intervenait dans les démarches que la Commission belge des Crimes de Guerre avait entreprises auprès des autorités américaines pour faire extrader vers la $B$ elgique le général $R$ eeder, chef de I'A dministration militaire pour la B elgique et le $\mathrm{N}$ ord de la $\mathrm{F}$ rance, en vue d'empêcher cette extradition. La justification avancée par l'auditeur général était analogue: la crainte que la venue de $R$ eeder ne paralyse toutes les instructions en matière d'épuration et de collaboration ${ }^{78}$. II n'obtiendra pas gain de cause et les deux hauts responsables allemands seront extradés et jugés en B elgique.

U ne des explications de l'attentisme du gouvernement, de l'absence totale d'intervention de Ganshof van der Meersch en matière de crimes de guerre, pourrait donc bien être son souci de préserver à tout prix le succès de la répression de la collaboration. R appelons que Ganshof arrive à $L$ ondres en juin 1943, qu'il entre en fonction en juillet et que le seul arrêtéloi pris en la matière par le gouvernement date du 5 août 1943. Plus aucune disposition ne sera adoptée par la suite.

\footnotetext{
77 UL B, P apiers Ganshof van der Meersch, 180P P/138, PV de la réunion du 20.09.1946.

$78 \mathrm{~V}$ oir à ce sujet $M$. B rébant, $L$ a collaboration internationale de la J ustice bel ge dans le cadre de la répressi on des crimes de guerre ( 1945-1948), mémoire de licence, UL B, 2007, pp. 85-88.
} 
Cette attitude de l'auditeur général, du gouvernement à $L$ ondres puis de ceux qui se succéderont après la Libération, gouvernants et parlementaires, Ganshof van der Meersch n'a pu ultérieurement en être vraiment satisfait. Quand, en juin 1947, la loi sur les crimes de guerre est votée, elle ne donne pas à la justice les outils adéquats pour pouvoir juger efficacement les criminels de guerre allemands. Les procès aboutiront à un échec, notamment en ce qui concerne les persécutions contre les J uifs, qui ne seront pas reconnues juridiquement, pas plus que les déportations ou le travail obligatoire ${ }^{79}$. Pour faire condamner les Allemands en raison de la politique antijuive qu'ils ont menée durant I'Occupation, I'auditeur géné ral n'entrevoit qu'une solution : I'application des articles du Code pénal relatifs aux infractions contre la Sûreté extérieure de l'É tat et plus particulièrement les articles 115 et 118bis du Code pénal.

D ans sa circulaire du 22 septembre 1947 consacrée à l'analyse de la loi du 20 juin 1947 relative à la compétence des juridictions militaires en matière de crimes de guerre, il s'adresse aux auditeurs militaires en ces termes: «II ne faut pas se cacher que seule l'application de ces articles pourra réprimer efficacement les actes de ceux qui ont exécuté ou participé personnellement à la politique générale de l'occupant dans l'un de ses aspects criminels: persécutions raciales, politique de déportations, mé thodes d'instruction barbares et inhumaines, pillage économique, etc. Cette politique criminelle générale, contraire aux lois et coutumes de la guerre, a eu pour conséquence directe la mort de milliers de Belges ou d'étrangers vivant en B elgique. Si I'on ne pouvait avoir recours à des articles de portée générale comme l'article 118bis du Code pénal, les responsables de cette politique ne pourraient être atteints en raison de I'inexistence actuelle d'un droit international intégré dans le droit pénal interne belge et sanctionnant de tel crimes. Une telle conséquence révolterait le sentiment et les exigences de la morale et de la justice [... ]. Quand le Chef de l'Allemagne et son gouvernement décrétèrent la déportation et ensuite l'assassinat en masse des J uifs de tous les pays occupés, ceux qui ont assuré l'exécution de tels crimes sur le territoire belge ne peuvent rester impunis. »80 Ganshof ne sera pas écouté, puisque la Cour de Cassation, dans un arrêt en date du 4 juillet 1949, considèrera que l'article 118bis postule la trahison, qui se définit comme un manquement au devoir de

\footnotetext{
79 V oir à ce sujet Crimes de guerre (J ugement des) , dans P. A ron - J . Gotovitch, op. cit., pp. 120-123. 80 CE GES, AA 1882, G/5-11-6, Circulaire nº 1903.
} 
fidélité envers I'É tat auquel, en principe, un sujet ennemi n'est pas tenu'81. Les responsables allemands des crimes commis contre les J uifs en Belgique ne seront donc pas punis à la mesure de ce qu'ils ont accompli. En sont responsables les hommes politiques belges de l'époque, ainsi que Ganshof van der Meersch, qui aurait dû défendre tous les arguments et considérations énoncés plus haut dès son arrivée à Londres. L'auditeur général ne semble pourtant pas insensible au sort des J uifs puisque, le 2 octobre 1944, dans le cadre de l'application aux étrangers de la circulaire $n^{\circ} 340$ sur l'internement administratif, il s'adresse au ministre de la J ustice pour remédier à une situation qui lui paraît pour le moins absurde : « V otre circulaire $n^{\circ} 340$, relative à l'internement administratif, stipule que les sujets allemands et les ressortissants d'États alliés de l'Allemagne doivent être internés [... ]. Or, dans certains cas, notamment dans celui des femmes qui, belges de naissance, ont épousé des réfugiés allemands israélites et ont subi la persécution de l'ennemi pendant I'Occupation, l'internement, même temporaire, au sortir des camps de concentration allemands paraît une mesure excessive, voire inhumaine. $\gg 82$

\section{Conclusion}

Dans le règlement par voie judiciaire des violations du droit de la guerre, le rôle des Belges sur le plan international revêt une certaine importance. Le plus actif d'entre eux est sans nul doute Marcel de Baer, qui occupe le poste de président de la commission $W$ ar Crimes à la L ondon I nternational A ssembly, au sein duquel il effectue un travail fort remarqué. Henri R olin participe aussi aux travaux de cet organisme. Or, de façon surprenante, leur travail et leur présence à l'échelon international ne se répercutent pas sur le plan national. Tous deux font partie du conseil législatif, organe chargé de préparer les textes de loi pour le gouvernement. E t pourtant nulle trace n'existe d'intervention de leur part qui serait en relation avec la problématique de la répression des crimes de guerre. Plus tard, en 1946, lorsqu'il s'agira de faire voter le projet de loi sur la compétence des juridictions en la matière, $\mathrm{H}$ enri R olin, rapporteur de ce projet pour le Sénat, fera preuve dans son argumentaire d'une certaine frilosité et rien

\footnotetext{
81 R evue de droit pénal et de criminologie (R DPC), 1948-1949, p. 986.

82 A GR, A rchives du H aut-Commissariat à la Sûreté de l'É tat, dossier n 1196, lettre de Ganshof van der Meersch au ministre de la J ustice, 2.10.1944.
} 
dans ses propos ne pourra laisser supposer une quel conque participation à l'élaboration des propositions novatrices de la London I nternational A ssembly, dont il fut pourtant le $D$ eputy $P$ resident.

En interne, ce sont surtout A ntoine Delfosse, ministre de la J ustice, et Charles Tschoffen, son chef de cabinet, qui interviennent en la matière. Walter Ganshof van der Meersch, censé être à la tête de la répression, n'entreprend rien de concret en ce qui concerne les crimes de guerre.

A lors qu'en novembre 1942 un document émanant du Premier ministre laisse entendre que la Belgique prendra toutes les mesures nécessaires pour pallier les insuffisances de la loi belge concernant les crimes de guerre, une seule disposition est prise : I'arrêtéloi du 5 août 1943 relatif aux faits commis par des étrangers hors du territoire belge. Celui-ci semble constituer à lui tout seul l'arsenal des mesures nécessaires. Or, dans ses interventions publiques, le ministre de la J ustice paraît très impliqué dans ce combat et il le sera d'autant plus que, trois mois après la fin de la guerre, il sera nommé président de la Commission belge des crimes de guerre, ce qui présuppose dans son chef un intérêt particulier pour le sujet.

Une des explications de l'immobilisme des autorités belges dans ce domaine est l'attente des décisions prises au niveau international : y aura-t-il ou non un code de droit international à intégrer dans le droit interne? L es autorités belges attendent-elles que les déportations, persécutions, mauvais traitements et prises d'otages soient incriminés au niveau international ? Quelle que soit la réponse, il ne fait guère de doute, en juin 1943, que c'est la loi nationale qui prévaudra et qu'il s'impose donc de préparer le travail, mais les mesures belges ne suivent pas.

Une autre explication est peut-être à chercher dans les relations du ministre de la J ustice avec l'auditeur général, lequel pèse de tout son poids, à l'instar des membres du gouvernement, pour donner priorité à la répression de la collaboration, tout en étant disposé à ce que la justice belge n'exerce pas ses prérogatives, et donc à risquer que les responsables allemands en B elgique ne soient même pas jugés par les tribunaux alliés ${ }^{83}$.

La volonté de s'en prendre particulièrement à ceux qui ont trahi plutôt qu'à l'ennemi lui-même ne constitue pas néanmoins une explication suffisante. Le travail sur ce plan ne présentait pas de difficultés majeures: l'arsenal juridique en matière de collaboration avec l'ennemi existait à la

83 Voir M. B rébant, op. cit. , p. 86. 
veille de la guerre et il suffisait de l'améliorer pour éviter d'être confronté à la difficulté visiblement insurmontable de porter atteinte au principe de la non-rétroactivité des lois.

Cette difficulté, si grave soit-elle, ne suffit pas à justifier que les autorités belges à Londres, alors qu'elles disposaient en décembre 1942 d'informations sur l'extermination des J uifs d'E urope et sur la situation dramatique que vivaient les J uifs de Belgique, n'anticipent en rien au niveau législatif. Dès le début 1943, elles auraient pu combler, fût-ce partiellement, le vide juridique existant et édicter un arrêtéloi portant sur les déportations, les persécutions et les mauvais traitements. La justice belge aurait pu alors, après la guerre, inculper les Allemands sur cette base, quand ce ne serait que pour les actes commis postérieurement à la date de la mise en vigueur de l'arrêté-loi. Ou encore, elles auraient pu, à l'instar des autorités françaises, procéder par voie d'interprétation. Au lieu de cela, la question des crimes commis à l'encontre des J uifs en B elgique, ainsi que celle de la sanction de leurs auteurs, est demeurée absente durant toute la guerre des préoccupations des autorités belges de $L$ ondres.

On peut espérer que les archives d'A ntoine Delfosse, si elles sont un jour accessibles au public, permettront de faire la lumière - en levant ainsi de nombreux motifs de perplexité - sur les manquements importants dont se sont rendues responsables les autorités belges de Londres lorsqu'elles préparaient le jugement des criminels de guerre allemands dans leur propre pays. 